\title{
TOWARDS SUSTAINABLE COMMERCIAL STREETS
}

\author{
Marwan Ali Fouad, \\ Institute of Graduate Studies and Research, Alexandria University, Alexandria, Egypt \\ Marwan@apgarche.ae
}

\begin{abstract}
The public space has always been the focus for many researchers, especially the controlling of the space through the urban configurations. The street space experience has been introduced as an approach to rethink in the public areas with a new method of thinking and how it affects the durability of streets to become sustainable within various dimensions of analytical studies. This Paper aims to set an approach for measuring and evaluate the state of Sustainability in the streets and public spaces through the previous studies done and extract six-different concepts within the three Sustainable dimensions that affect the street users which are (design configurations, activity and Environmental aspects). Moreover these criteria can highlight the commercial street problems in the Egyptian Streets and determine the strong and weakness points through creating an Evaluation Reference set through harmonizing the concepts with the Dimension approach and apply this reference set on the streets for measuring state of sustainability. All this result would help to set recommendations for better public spaces and protect the city character, enhance the streets that characterize the city, neglecting all the random unorganized activities that affect directly the pedestrians, and finally enhancing the controlling of the sustainable public space. on the other hand the paper also works to find a set of scientific recommendations for planning and the need to take into account during the process of urban design with the integration of the environment, a basic standard to be based on environmental and design criteria without affecting the resources and needs of future generations.
\end{abstract}

Keywords: Commercial streets, sustainable streets, Environmental Aspects, Street activity, Accessibility, Pedestrians, Place identity.

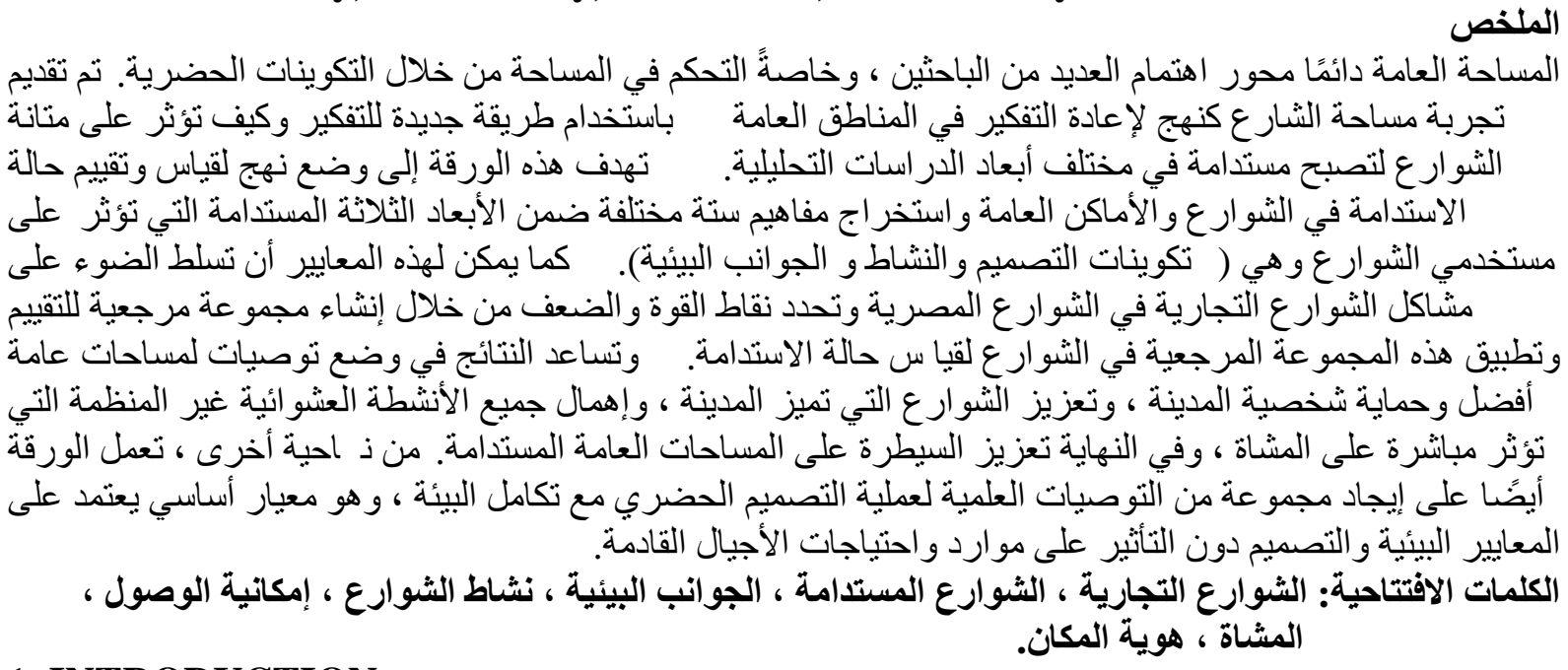

\section{INTRODUCTION}

The public space is changing. Pedestrians are now living in a world with a negative environment, streets radically different. These new spaces vary in quality and intensity from 
those of the past; however, many researchers have been alerted to the dangers of the random spread of loss in control that lead to informal streets into every Public Space.

Every Public space has a spatial character which affected by proportion, scale, size of parts relative to each other, color, texture, furnishing and finally its activities. We need to develop an appropriate understanding of them and to treat them as dynamic physical objects with a multi-dimensional element, (Gontier, 2006).More Over the public space must link the main public urban space pillars which are the Life activities of the users in the space then the Space place which took place within the area and the surrounding Buildings that will enclose the space around it to generate the whole Public Space Image (Gontier, 2006).

A Street that is always alive with people and movement is an important and significant street, rather than a dead one where no one passes or not used frequently. Moreover, the more the street becomes full of people, the more people feel safer with the presence of the others. The job of handling strangers mostly depends on the streets of a city (J. Jacobs, 1961).

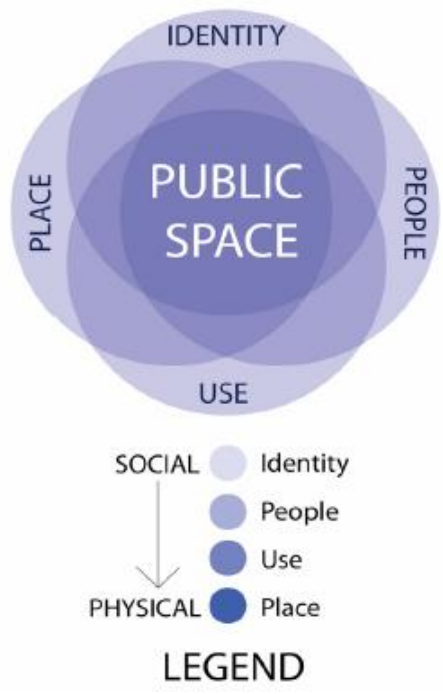

Figure 1: Public Space Characteristics Source: Brcelona Council 2004

Commercial streets are the Areas in the city that intended to provide a variety of Shopping, activities, and service options and convenience. (Planning council, 2012) The commercial streets primarily c0mposed of commercial building or shops Where the Commercial activity took place as the a downtown, central business district, financial district, "Main Street", commercial strip, or shopping center includes the buying and selling of goods and services in retail businesses, While commercial activities typically take up a relatively small amount of land, that are enhancing the community economy.

According to (JONES, 2008) Urban commercial streets provide the setting for a wide range of urban street activities, which can be grouped under two street functions of 'Place' and 'Accessible Link'.

As an Accessible Link, a street provides an accessible tube for movement, and forms an integral part of the wider urban street network and other, more specialized, urban transport networks, A Link user may travel by a variety of modes, from private car or truck to bus, bicycle or on foot. Their essential need is to follow a continuous, linear path through the street network, with minimum disruption and a seamless connection from the beginning to the end of their journey. In general, they are seeking to minimize travel time along each section of street.

As a Place, Urban Commercial Street is a destination in its own right: a location where activities occur on or adjacent to the street. A Place user is someone wishing to make use of some of the features that are on that particular street, and will usually do so on foot. While such people are classified as 'pedestrians', they are not passing through the area - they are spending time in the area, and may be carrying out a wide variety of activities (e.g. shopping, 
talking, waiting, resting, working). They are particularly affected by the noise and air pollution produced by vehicular traffic, and the general severance effect of heavy traffic volumes in inhibiting their movement between places on opposite sides of the street. However, not all of the traffic and transport-related activity observed on urban streets is part of that street.

\section{Sustainable Streets Strategy}

In order to convert the Traditional Negative Streets into an effective positive Streets with activities and Design a place that can serve the current generations without affecting the new generations it should be developed on a Sustainable strategy way which can be named as a sustainable Development Plan but before that we should Review the sustainability and Its Main Pillars that affect the Urban Space.

A generally accepted definition of sustainable development, and a good point to begin an exploration of this concept, is taken from the Brundtland Report: 'Sustainable development is development that meets the needs of the present generation without compromising the ability of future generations to meet their own needs' (World Commission on Environment and Development, 1987).

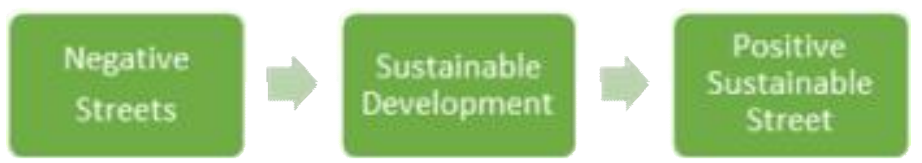

Figure 2: Sustainable Street Strategy Through Sustainable Development Source: Author.

All the Previous Studies that studied the Sustainable Streets in the field of Urban Design and Sustainability Basic Pillars linked them to certain Concepts that are important and necessary for judgment and results. In achieving the sustainable streets and control them several theorists literally linked Sustainable streets to certain concepts (Lukes, 2008) (Anon., 2006) (Jacobs, 1993) (Lynch, 1960) and according to all those researchers negotiate the management strategy for the Commercial Streets that can be listed in the below Points:

- Make a unique local identity and civic pride

- Shape the existing and future development and

- Enhance the physical character of an area,

- Improve the social integration, including accessibility

- Provide connected routes between Street nodes recreation, walking and cycling, and safer routes Schools.

- Protect and enhance the biodiversity.

- Re-provide green infrastructure and ecosystem Services.

- Increase and boost the potential of shopping, which will increase the economical income. and Cultural activities provide cultural, social, recreational, sporting and community facilities

- Promote understanding of the historical, cultural and archaeological value of place identity.

- Contribute to the create a safe and healthy place, Including quiet areas

- Provide popular outdoor commercial facilities

- Help mitigate and adapt to climate change

- Improve environmental opportunities to enjoy contact with the natural world.

This illustrates the Necessity of the Presence of all those studies and Concepts in order to understand the Sustainable Streets. A referential Set must be exported from a big Number of literature and theoretical Previous Studies in order to deduce this set of Concepts.

Six Main Concepts were extracted from theoretical Reference, Each Concept was studied by theorists in order to gain indicators that could be used to recognize the presence of such a concept. 
Table 1: Main Theoretical Concept extracted from Previous Studies source: Developed by Author.

\begin{tabular}{|c|c|c|}
\hline Concept & Example & $\begin{array}{l}\text { Theoretical } \\
\text { Reference }\end{array}$ \\
\hline A. Place Identity & $\begin{array}{l}\text { "Iconic Streets Imbued with a lasting Image and Grounded } \\
\text { sense of place is Essential Component to all Urban and rural } \\
\text { Cities. Not all streets can have a city-wide Iconic Identify. } \\
\text { However, some Streets take on that role of telling the story of the } \\
\text { area and the larger region it represents. " }\end{array}$ & $\begin{array}{l}\text { (Anon., 2006) } \\
\text { (Hague, 2005) } \\
\text { (Jacobs, 1993) } \\
\text { (Harmouth 1990) } \\
\text { (Proshansky, 1978) }\end{array}$ \\
\hline $\begin{array}{l}\text { B. Mixed of Uses } \\
\text { (Activity) }\end{array}$ & $\begin{array}{l}\text { "Activities are the basic Building Blocks of any place. Having } \\
\text { something to do gives people a reason to come to a place -and } \\
\text { Return. when there is nothing to do, a space will be empty and } \\
\text { that generally Means that something is wrong " }\end{array}$ & $\begin{array}{l}\text { (Petersen 2006) } \\
\text { (Brtonetal ,2003) } \\
\text { (Gtz1999) } \\
\text { (Tibbalds ,1992) }\end{array}$ \\
\hline $\begin{array}{ll}\text { C. } & \begin{array}{l}\text { Pedestrian } \\
\text { Environment }\end{array}\end{array}$ & $\begin{array}{l}\text { "Pedestrian life streets are full of life that enhance pedestrian } \\
\text { safety and mobility for all the users of the street." }\end{array}$ & $\begin{array}{l}\text { (Elhamy, 2012) } \\
\text { (Evans, 2009) } \\
\text { (Senbel,2008) } \\
\text { (Bentley, 1985) } \\
\text { (Gehl ,1989) }\end{array}$ \\
\hline D. Accessibility & $\begin{array}{l}\text { "Building an active Places and path ways of Streets for People, } \\
\text { they Need them to be accessible. when they are not we are } \\
\text { excluding people from participating in the social or economic } \\
\text { activity that is going on in that space" }\end{array}$ & $\begin{array}{l}\text { (Lynch, 1960) } \\
\text { (Jacobs, 1961) } \\
\text { (Gehl ,1989) }\end{array}$ \\
\hline E. Safety & $\begin{array}{l}\text { "when People say that a city, or a part of it, is dangerous or is a } \\
\text { jungle what they mean primarily is that they do not feel safe on } \\
\text { the sidewalks" }\end{array}$ & $\begin{array}{l}\text { (Lyshan 2014) } \\
\text { (Evans, 2009) } \\
\text { (Jacobs, 1961) } \\
\text { (Mslow 1954) }\end{array}$ \\
\hline $\begin{array}{l}\text { F. Green } \\
\text { Environmental } \\
\text { Aspects }\end{array}$ & $\begin{array}{l}\text { "Effectively incorporating green techniques into the Street } \\
\text { network provides significant opportunity to decrease } \\
\text { infrastructure demands and pollutant transport and change the } \\
\text { street Life" }\end{array}$ & $\begin{array}{l}\text { (PoolR., 2009) } \\
\text { (Ryser J.2009) } \\
\text { United Nations -Human } \\
\text { Settlement 2009) }\end{array}$ \\
\hline
\end{tabular}

\section{DIMENSIONS OF SUSTAINABLE COMMERCIAL STREETS}

As a result, from the Previous Studies for the Existence of the Sustainability in the Urban Streets can be easily recognized when taking in consideration the three Sustainable Pillars (social. Economic and Environmental) Pillars with the previous Studies of (Jacobs, 1993) (Greenberg, 2009), (Lynch, 1960) (lehmann, 2010) and (Jones, 2008) in the concepts of successful green Street.

\section{Design Configurations Dimensions}

Create A network that supports Communities and places

Integrate the streets with the natural Different Scales systems

Respect the Current Natural Existing

Environment

Mixed Land use activity.

Pedestrian opportunity.

Variety of transportations. (Maximize

Transportations Facilities).

\section{Activity Dimensions}

Increase the social

Interaction.

Pedestrian Activity.

Welcoming interactivity.

Enhancing Security and safety.

Encouraging Community

Participation.

\section{Environmental Dimensions}

Reduce impacts on

environmental resources.

Reduce energy consumption.

Improve rainwater reuse quality.

Recycling Methods.

Material selection For Streets

and Pavements.

Figure 3: three sustainable public space dimensions Source: Developed by Author.

The Relation between the users of the street to the urban space can be affected by the Activity that take place in the street, also affected by the Design configurations that are managed and planned to fit all the user needs to give them a comfort safe place to make their activities within a well Environmental Zone surround all the street elements to reach the target of sustainability in the street space. 
Therefore the sustainable streets were linked in the theory with the six concepts: place identity mixed of uses, pedestrian environment, accessibility, safety and environmental aspects. These Concepts were Very Important for deducing the Sustainability Of every Street, the link was stated directly or in directly in the previous Studies. Each of the six Concepts Expressed and linked to the three concluded Sustainable Dimension: Design, Activity and Environmental Dimensions to extract the Criteria of the Sustainable Commercial Streets.

The six Concepts were found to have an important role in theory which requires their integration Together.

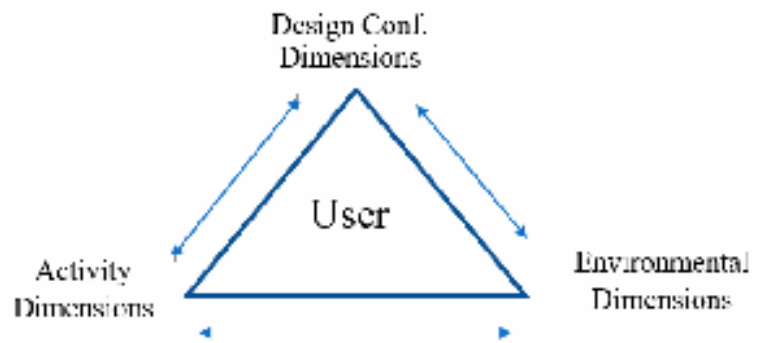

Figure 4: the relation between the street user and the Three Dimensions, source: Author.

\section{CONCEPT ANALYSIS METHODOLOGY}

The main methodology is to study each concept separately across the three dimensional pillars through the Previous Studies and generate the main elements for each concept to set the street actual sustainable elements.

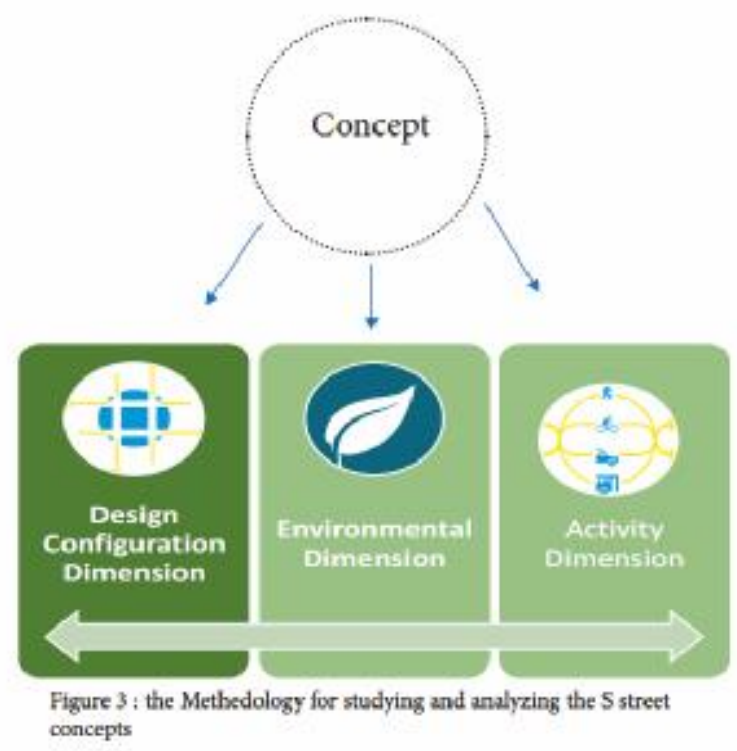

\subsection{Place Identity as a Concept:}

Lately, people have been talking increasingly about "loss of the place identity." What they're missing is the kind of place that has been special in their lives and given them a strong sense of community and well-known character for the surrounding Urban space elements that share a known unique character.

Iconic Streets Imbued with a lasting Image and Grounded sense of place is Essential Component to all Urban and rural Cities. Not all streets can have a city-wide Iconic Identify. However, some Streets take on that role of telling the story of the area and the larger region it represents. (Anon., 2006)

The Place identity or image of the place and in some ways it is related to the concepts of Place attachment and Sense of place. Refers to a cluster of ideas about place and identity in 
the fields of geography, urban planning, urban design, landscape architecture, environmental psychology and urban /ecological sociology. It concerns the meaning and significance of places for their inhabitants and users, and how these meanings contribute to individuals' conceptualizations of self. (Hague, 2005).

According to (Realm, 2016) image and identity street types can be supported and facilitated through the design process of the street environment and been divided according to the scale, facilities,

Activities and the street historical background, First Downtown main streets, as the original historic commercial mixed-use traffic street continue to be a major thoroughfare for the city, but also function as an important work center, public gathering space, administrative core and shopping destination. Clearly marked and signaled crosswalks and intersections, high quality furnishings and streetscape plantings.

Hormuth (1990) discusses the role of relocation and self-concept change suggesting that choosing to move can represent self-concept change with the old place becoming a symbol of the old self and the new place representing an opportunity to develop new identities. In both these examples place is considered to be an active part of the construction of a person's identity, representing continuity and change.

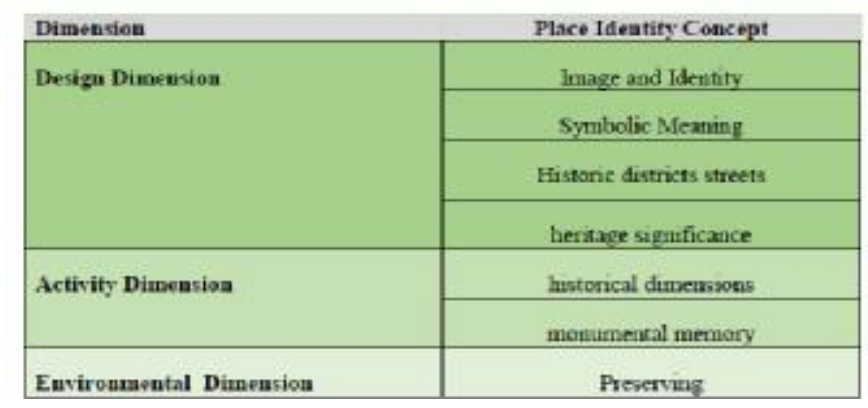

\subsection{Pedestrian Environment as a Concept}

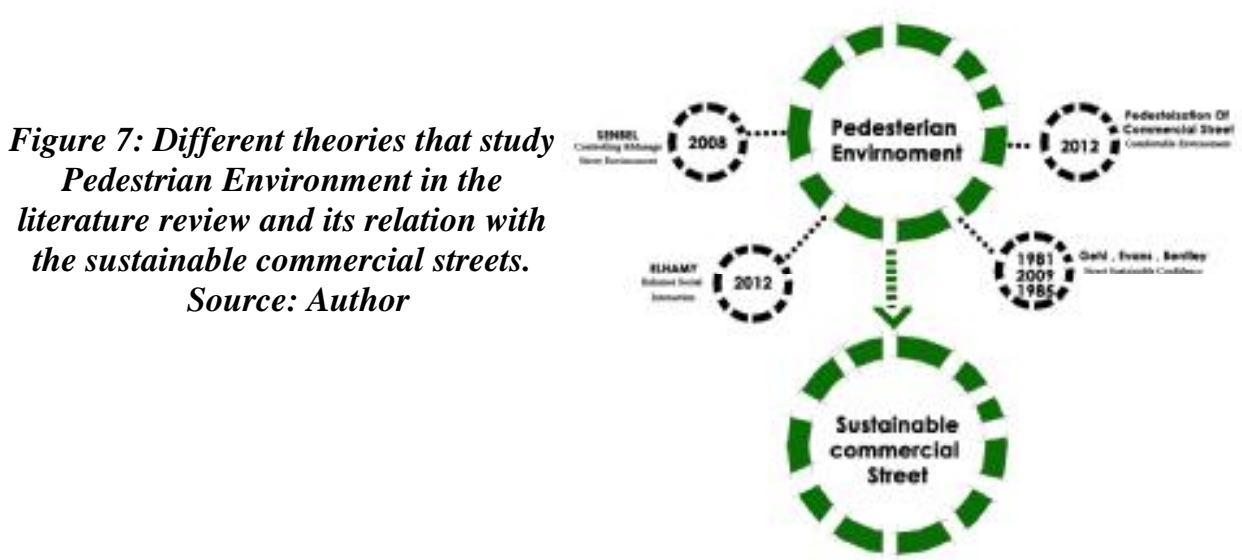

Pedestrian environment (also known as auto-free zones and car-free zones) are zones of a city or town reserved for pedestrian use and in which

All motorized traffic is prohibited. Some streets are partially pedestrianized, for instance during certain times such as evenings or weekends, while other, pedestrian-priority streets use Traffic Calming design strategies to control traffic speeds and volumes (Boyd 1998). According to (Elhamy, 2012) the pedestrian life Streets Are full of Life that enhance pedestrian design. These components convert the pedestrian life network in urban and suburban areas in to active well-designed commercial streets. Paving, street furniture landscaping and lighting make the public sidewalk a place where people want to participate and enhance 
the social interaction. These features create a sense of place identity on the street and are also important visual traffic calming measures. Furthermore, retail and restaurants increase the approach of pedestrian-friendly streets.

People also want to have their shopping in an environment where they can feel safe, not only safe from motor vehicle traffic, but safe from crime or other concerns that can affect personal security. Areas need to be well lit to encourage walking during evening hours. If the pedestrian system is not accessible, it is often not safe. For example, lack of access may cause wheelchair users to use the street rather than a poorly maintained sidewalk. Some populations may be at a higher risk of pedestrian crashes. Children under age 15 are the most overrepresented group in pedestrian crashes and people over age 65 have the most pedestrian Facilities,

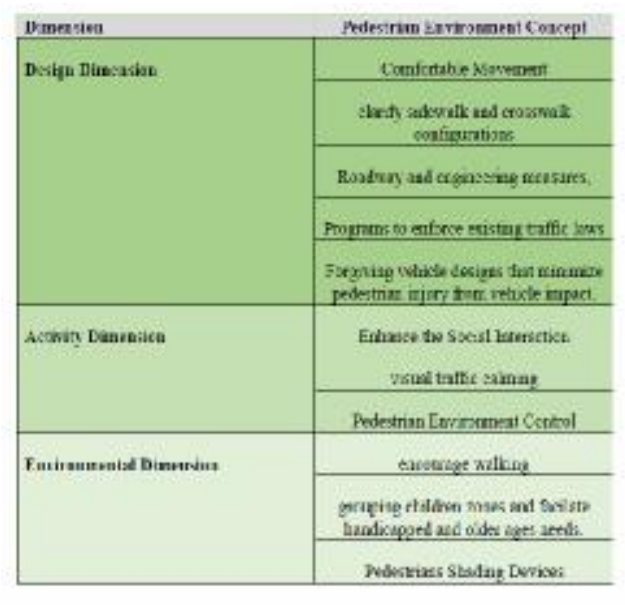

Therefore, it is especially important to provide facilities for grouping children zones and facilitate handicapped and older ages neers

2.3 Mixed of Uses as a concept

Figure 8: Different theories that study Mixed of uses in the literature

review and its relation with the sustainable commercial streets . Source: Author

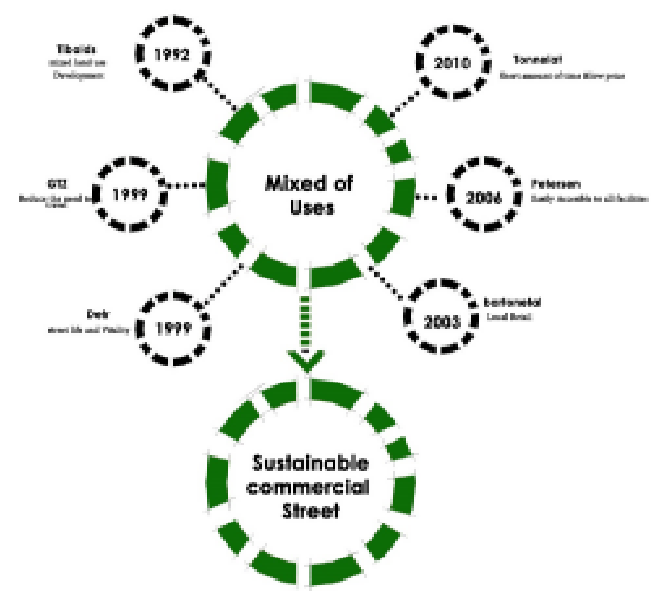

Many urban designers now promote the advantages of traditional mixed-used streets. This has forced a consideration of how such streets might be designed and how they shape the urban fabric. According to Marshall $(2005 ; 2009)$ the urban structure, understood in a purely physical sense, is highly influenced by the structure of movement and access. The connective tissue (Marshall, 2005) of urban space is identified here with movement to and through spaces. An urban flux that carries social and cultural meaning where street patterns are the production of the geometry of movement and connectivity of places.

While the sustainable UK Labour government strategy operates at a broad scale. At the local level, definitions of sustainability are harder to derive within the relatively limited context of 
a mixed-use street. Barton et al (2003), in their manual for new sustainable neighborhoods, propose a reinvention of the mixed-use high street. Their argument for its sustainability as a focus for local retail and commercial uses is based on the catchment area for a residential neighborhood enabling local residents to walk or cycle to the facilities. This provision of local services that are accessible by pedestrians Sustainability objectives.

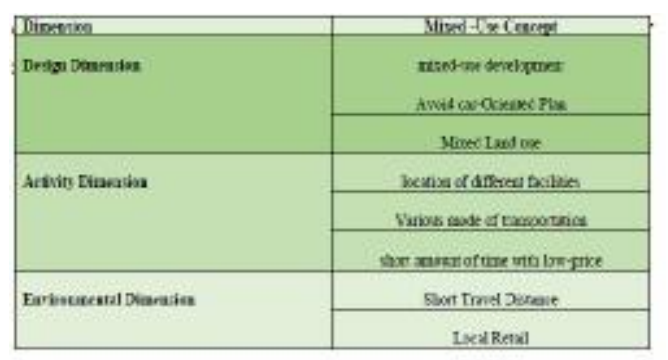

\subsection{Accessibility as a Concept}

Figure 9 : Different theories that study Accessibility in the literature review and its relation with the sustainable commercial streets .

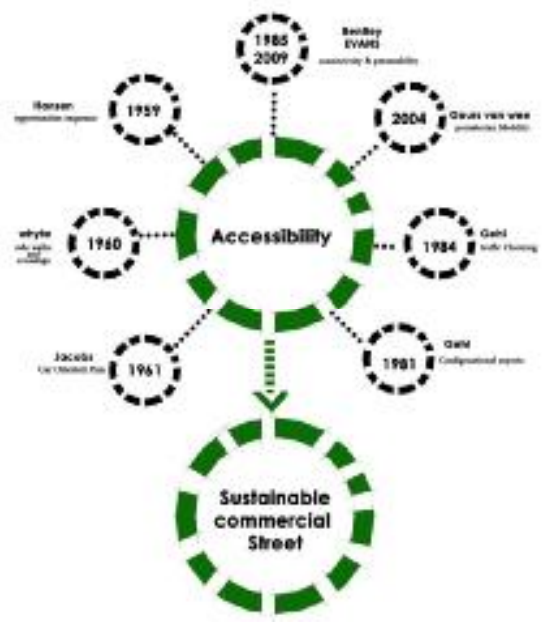

Accessibility as a concept was studied in terms of vehicle movement. So, it was transportation activity that was mainly considered (Evans2009), which overlooked accessibility in favor of car mobility and ignored its true meaning, as such design focused on cars and their movement which led to unpleasant chaotic streets. Consequently, a new paradigm shift was required to link accessibility to its original meaning and the people centered approach rather than the carcentered approach appeared (floater,2014).

(Jacobs, 1961) Described all the surrounding streets design was based on a concept of "Car Oriented Plan" which is based on the car proportions, size and active space which affect all the street width and sidewalks in the planning Design process to fit all the car needs and this affect the cross distance and change the suitable distance for the pedestrians' users that directly affect the distance, time and Efforts that led to the decreasing of "pedestrian Mobility "in our streets. This mean that Accessibility term should be designed to serve all various users with different needs and to study the pedestrian, handicapped, children. Elder age users' needs to reach their target in the shortest distance and limited period of time without the less obstacles.

The Pedestrian Mobility as (Geurs, 2004) Studied that it is one of the accessible physical Design Dimension. Having Defined Nodes across the street is a main need for accessibility. They Guarantee the Recharging of Pedestrians ability, to continue their journey through the street. (Geurs, 2004) also, that Effort and time are important needed, the more the commercial street is accessible. (patxi, 2015) works on raising the individual's confidence in using the commercial street through Defining lanes and presence of Directional Signs. 
The space accessibility would be more dynamic and safe along the same street directly movement in a commercial street for an example the distribution of the retail stores and the user would reach them in an easy way without any segregation External Elements. This would Force the users to place various and different activities either a commercial activity or even create a new approach for more and more new activities in the street within different scale of street either intimate or large scale for the Urban space. Moreover the site location of the urban elements and buildings should be selected and designed in such a manner that creates no obstacles and distortion for the view of residents of the surrounding buildings.

Figure 10 : Different theories that

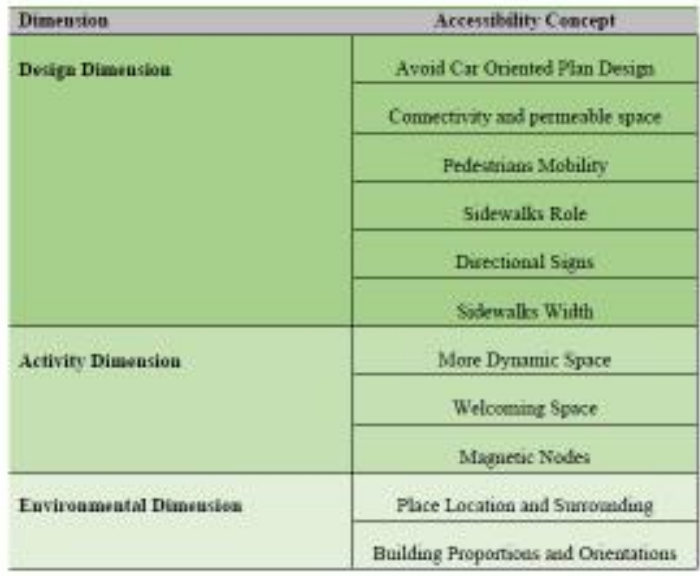
study Safety in the literature review and its relation with the sustainable commercial streets .

\subsection{Safety as a Concept}

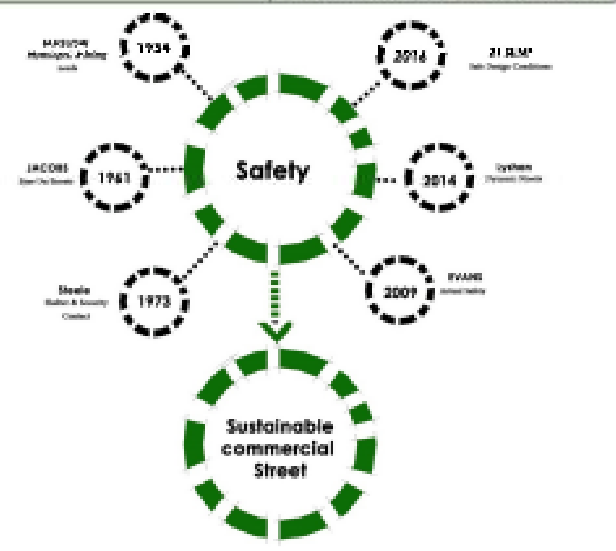

Safety is a substantial need in commercial streets where its absence prevents the occurrence of a dynamic life of the street and keeps the street far from achieving high standards. (Lyshan,2014),An empty street for the users is like an empty painting frame, dangerous Space for users . Such surveillance requires a big number of stores and public places scattered along the sidewalk; especially night used places such as shops and stores that contribute to the safety and security, these sidewalks gain actual reasons for its usage by both the residents and the random users of these enterprises.

The role of natural day light is highly important in the street safety, Bright street lights gain extra value for the comfort and safety they offer to people, therefore increasing the contribution of these people as a surveillance eye on the street. However, streetlights cannot perform on its own. Shocking crimes still occur in well-lighted stations and places. And unless there are eyes that overlooks these places holding the unconscious assurance of street support and safety these crimes will still occur (J. Jacobs, 1961). 

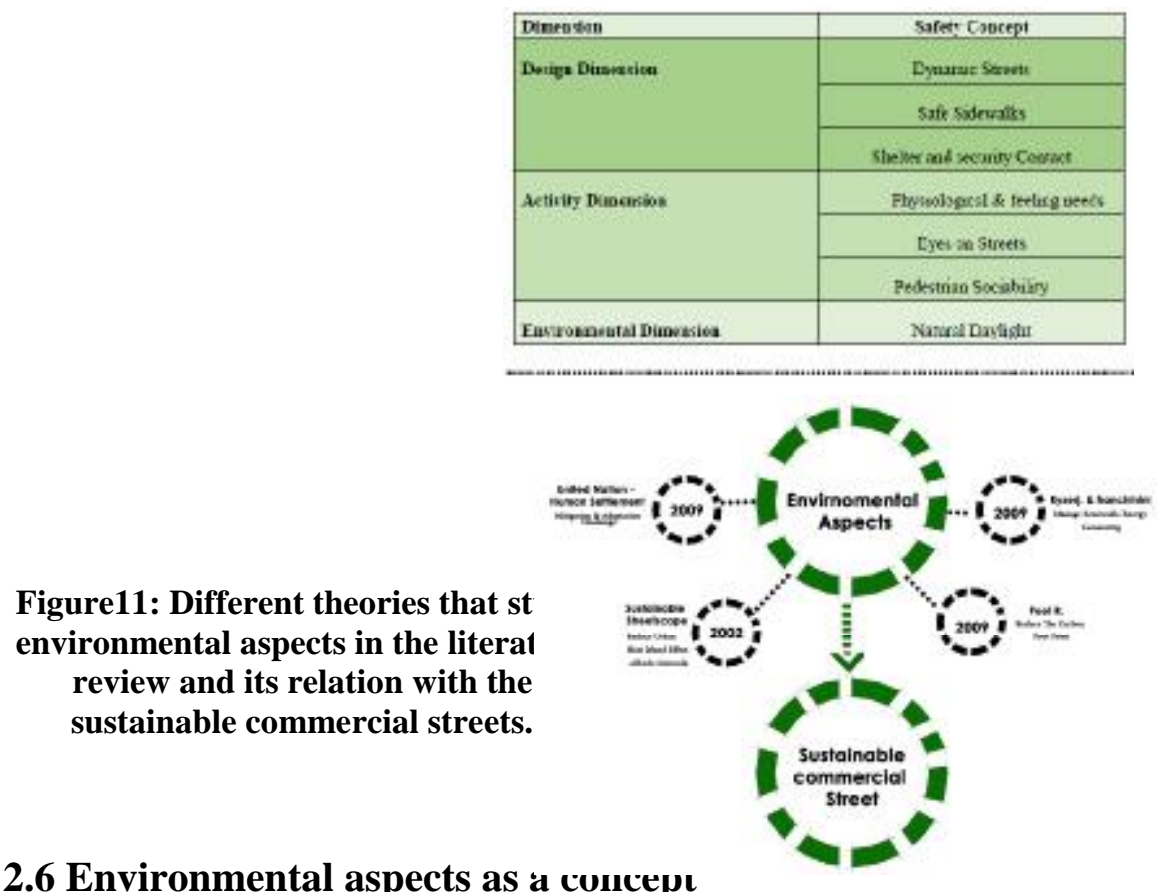

\subsection{Environmental aspects as à concept}

In our Community and Urban Spaces Environment and energy are two sides of the same coin. The Streets are the major consumers of fossil fuels which have risen from $4 \%$ of global energy consumption in the early 1900 s to over $86 \%$ at present time. For all these reasons, Streets play an excessive role in greenhouse gas emissions. Mitigating $\mathrm{CO} 2$ emissions and adapting to climate change are essential at the scale of a street level. in Egypt consumes $19.2 \%$ of the total energy consumption and about $39.1 \%$ of the total electricity consumption, which produces $10 \%$ of the total CO2 emission, (Ministry of Energy and Electricity, 2003). On the other hand, Egypt has a good potential of renewable resources including solar and wind power. Thus, renewable energy technologies can be considered as an integral part of the Egyptian energy policy framework. (Gihan Mosaad Hannallah, 2014).

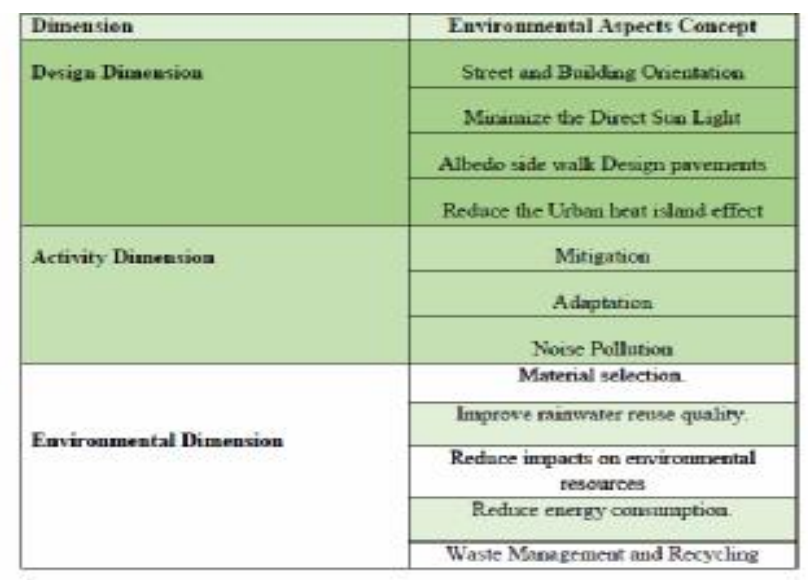

\subsection{Extracting Concepts:}

This Extracting Concepts and Dimensions formulates an accumulative meaning of sustainability in the commercial streets. Which is the afordance of the physical active design within the surrounding environmental to attract and support Pedestrians activities accompanied by providing investments and economic Growth. 
Theorists who studied Sustainability through time linked the state of sustainability to complicated Concepts. The research arrived at the six concluded concepts that repeated by theorists through different eras in their study of the sustainability in the streets, it become clearer through the analytical examples that all the six concepts must be achieved at different various levels, towards the state of sustainability target

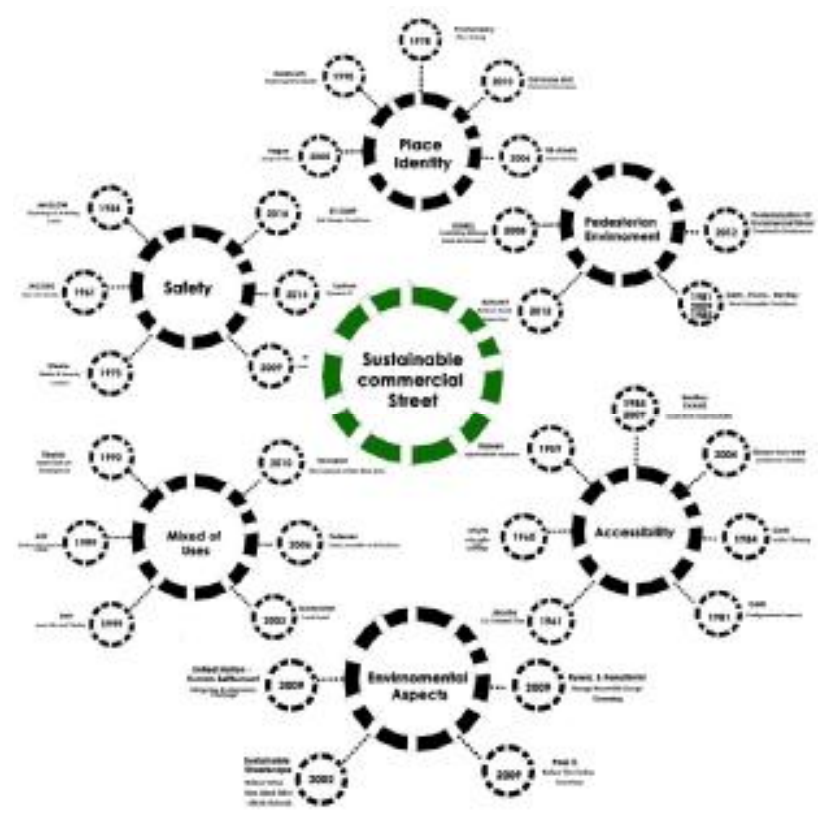

Figure 12 : link between the six concepts of sustainable commercial streets

Later, the research considered these six concepts as the Concepts of Sustainable Commercial Streets. As they all imperative to reach the state of sustainability, they are: place identity, Mixed of Uses, Pedestrian Environment, Accessibility, Safety and The Environmental Aspects. Coordinating each concept's indicator in design, activity and Environmental Dimensions shaped the Reference table. This table is set into two axial. It consists horizontally from the six concepts and vertically from the three dimension. 


\begin{tabular}{|c|c|c|c|c|c|c|}
\hline \multicolumn{7}{|c|}{ Concepts of Sustainable Commercial Streets } \\
\hline Dim. & Place Identity & $\begin{array}{c}\text { Mixed } \\
\text { Uses } \\
\end{array}$ & $\begin{array}{c}\text { Pedestrian } \\
\text { Environment }\end{array}$ & Accessibility & Safety & $\begin{array}{c}\text { Environmental } \\
\text { aspects }\end{array}$ \\
\hline \multirow{6}{*}{ 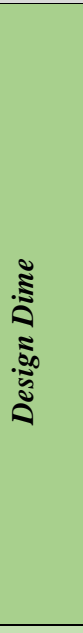 } & $\begin{array}{l}\text { Image and } \\
\text { Identity }\end{array}$ & $\begin{array}{c}\text { Mixed-use } \\
\text { development }\end{array}$ & $\begin{array}{c}\text { Comfortable } \\
\text { Movement }\end{array}$ & $\begin{array}{c}\text { Avoid Car } \\
\text { Oriented Plan } \\
\text { Design }\end{array}$ & $\begin{array}{c}\text { Dynamic } \\
\text { Streets }\end{array}$ & $\begin{array}{l}\text { Street and Building } \\
\text { Orientation }\end{array}$ \\
\hline & $\begin{array}{l}\text { Symbolic } \\
\text { Meaning }\end{array}$ & $\begin{array}{l}\text { Avoid car- } \\
\text { Oriented } \\
\text { Plan }\end{array}$ & $\begin{array}{c}\text { Clarify sidewalk and } \\
\text { crosswalk } \\
\text { configurations }\end{array}$ & $\begin{array}{c}\text { Connectivity } \\
\text { and permeable } \\
\text { space }\end{array}$ & $\begin{array}{c}\text { Safe } \\
\text { Sidewalks }\end{array}$ & $\begin{array}{c}\text { Minimize the Direct } \\
\text { Sun Light }\end{array}$ \\
\hline & $\begin{array}{c}\text { Historic } \\
\text { districts } \\
\text { streets }\end{array}$ & $\begin{array}{l}\text { Mixed Land } \\
\text { use }\end{array}$ & $\begin{array}{l}\text { Roadway and } \\
\text { engineering } \\
\text { measures, }\end{array}$ & $\begin{array}{l}\text { Pedestrians } \\
\text { Mobility }\end{array}$ & $\begin{array}{l}\text { Shelter and } \\
\text { security } \\
\text { Contact }\end{array}$ & $\begin{array}{l}\text { Albedo side walk } \\
\text { Design pavements }\end{array}$ \\
\hline & $\begin{array}{l}\text { heritage } \\
\text { significance }\end{array}$ & & $\begin{array}{l}\text { Programs to enforce } \\
\text { existing traffic laws }\end{array}$ & Sidewalks Role & & $\begin{array}{l}\text { Reduce the Urban } \\
\text { heat island effect }\end{array}$ \\
\hline & & & $\begin{array}{c}\text { Forgiving vehicle } \\
\text { designs that } \\
\text { minimize pedestrian } \\
\text { injury } \\
\end{array}$ & $\begin{array}{l}\text { Directional } \\
\text { Signs }\end{array}$ & & \\
\hline & & & & $\begin{array}{l}\text { Sidewalks } \\
\text { Width }\end{array}$ & & \\
\hline \multirow{3}{*}{ 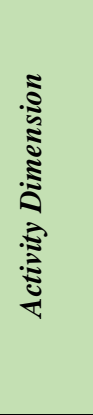 } & $\begin{array}{c}\text { Historical } \\
\text { dimensions }\end{array}$ & $\begin{array}{l}\text { location of } \\
\text { different } \\
\text { facilities }\end{array}$ & $\begin{array}{l}\text { Enhance the Social } \\
\text { Interaction }\end{array}$ & $\begin{array}{l}\text { More Dynamic } \\
\text { Space }\end{array}$ & $\begin{array}{l}\text { Physiologic } \\
\text { al \& } \\
\text { feeling } \\
\text { needs } \\
\end{array}$ & Mitigation \\
\hline & $\begin{array}{c}\text { Monumental } \\
\text { memory }\end{array}$ & $\begin{array}{c}\text { Various mode } \\
\text { of } \\
\text { transportatio } \\
n \\
\end{array}$ & $\begin{array}{l}\text { visual traffic } \\
\text { calming }\end{array}$ & $\begin{array}{l}\text { Welcoming } \\
\text { Space }\end{array}$ & $\begin{array}{c}\text { Eyes on } \\
\text { Streets }\end{array}$ & Adaptation \\
\hline & & $\begin{array}{c}\text { short amount } \\
\text { of time with } \\
\text { low-price }\end{array}$ & $\begin{array}{c}\text { Pedestrian } \\
\text { Environment } \\
\text { Control }\end{array}$ & Magnetic Nodes & $\begin{array}{l}\text { Pedestrian } \\
\text { Sociability }\end{array}$ & Nosie Pollution \\
\hline \multirow{5}{*}{ 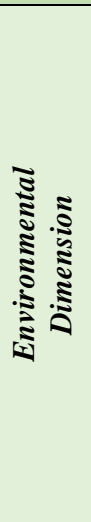 } & Preserving & $\begin{array}{l}\text { Short Travel } \\
\text { Distance }\end{array}$ & Encourage walking & $\begin{array}{c}\text { Place Location } \\
\text { and } \\
\text { Surrounding }\end{array}$ & $\begin{array}{l}\text { Natural } \\
\text { Daylight }\end{array}$ & Material selection. \\
\hline & & Local Retail & $\begin{array}{l}\text { Grouping children } \\
\text { zones and facilitate } \\
\text { handicapped and } \\
\text { older ages needs }\end{array}$ & $\begin{array}{c}\text { Building } \\
\text { Proportions and } \\
\text { Orientations }\end{array}$ & & $\begin{array}{l}\text { Improve rainwater } \\
\text { reuse quality. }\end{array}$ \\
\hline & & & $\begin{array}{c}\text { Pedestrians Shading } \\
\text { Devices }\end{array}$ & & & $\begin{array}{c}\text { Reduce impacts on } \\
\text { environmental } \\
\text { resources }\end{array}$ \\
\hline & & & & & & $\begin{array}{l}\text { Reduce energy } \\
\text { consumption. }\end{array}$ \\
\hline & & & & & & $\begin{array}{c}\text { Waste Management } \\
\text { and Recycling }\end{array}$ \\
\hline
\end{tabular}

\section{SUSTAINABLE COMMERCIAL STREET IN PRACTICE (TESTING METHODOLOGY)}

This Analysis on Two Commercial Streets, an international example and a local one, their Aim are to examine the appliance of the Six Developed in the previous section. the need of the state of sustainability in the Commercial Streets through the six concepts together to achieve the state of sustainability. Two steps were taken in consideration in analyzing the two examples. First, illustrates the Reasons of being in the state of Sustainability. Secondly, Determines the Concepts Each Street Followed to provide the State of sustainability through the indicators Design, Activity and Environmental Dimensions for the Commercial Streets.

\subsection{First Examine Case: King-Victoria Transit Hub Street.}

The King-Victoria Transit Hub, is known as the Central intermodal transportation Station, terminal in Kitchener, Ontario, Canada. It is a planned as the It will serve the area of Ion light rail line, GO Transit trains and Via Rail. The station will replace the current Kitchener railway station and the 
intercity bus services which currently depart from Charles Street will be relocated to the new proposed new terminal.

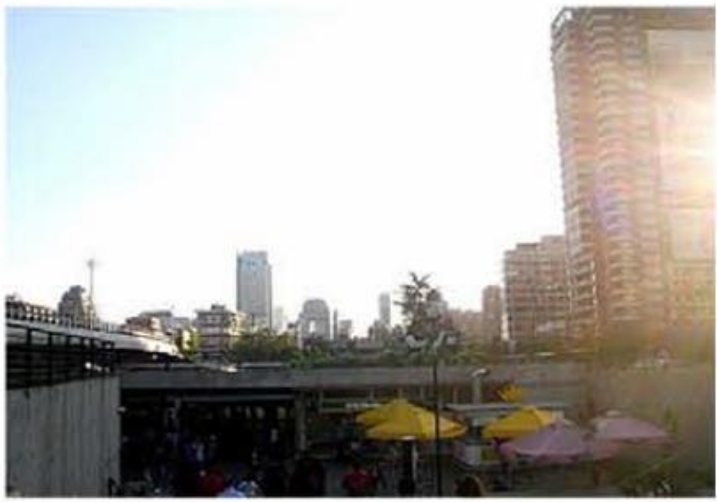

Figure 1 the day light gives the underground space safe feeling and natural day light

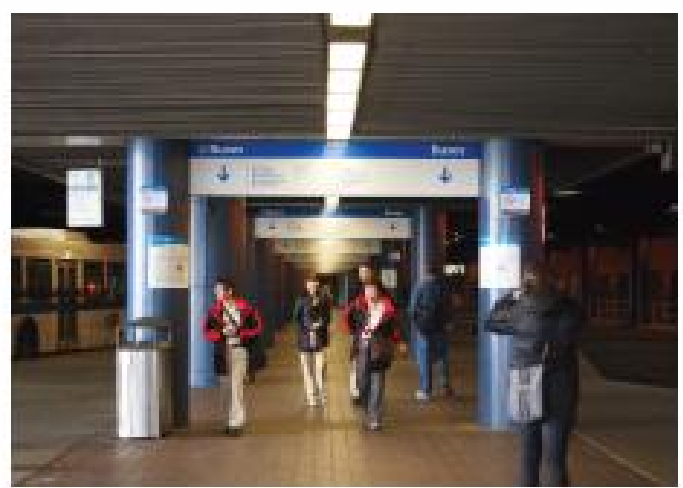

An off-street bus terminal that serves intercity buses, GO Transit buses, and GRT buses that terminate at the Transit Hub. The terminal could also allow for MobilityPLUS pick-ups and drop-offs

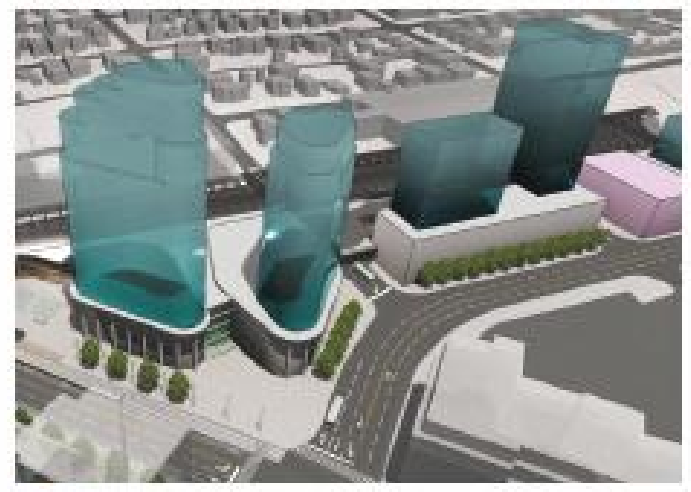

Mixed-use development integrated with the Transit Hub, with office, retail, residential, and other uses at a higher intensity forming a landmark in downtown Kitchener

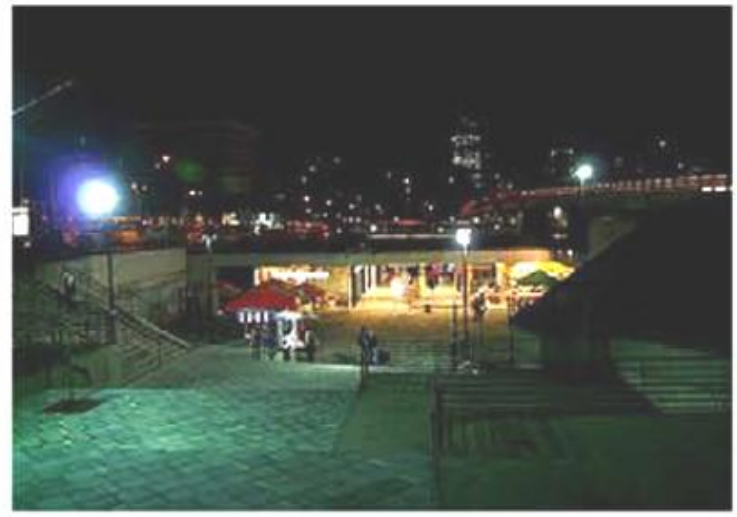

Figure 2 also the night commercial light gives the same safe feeling space

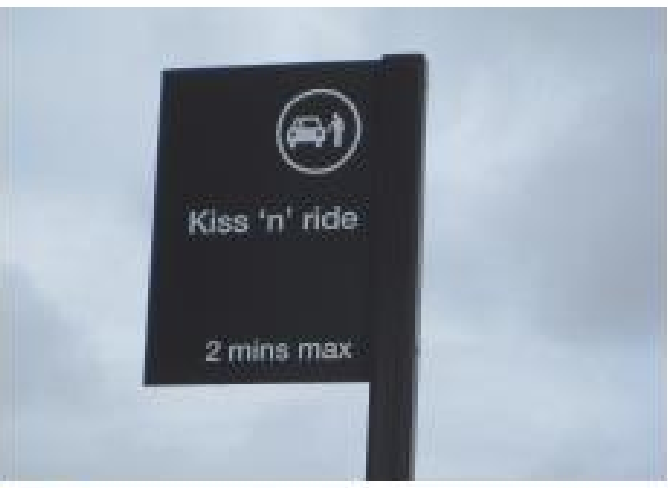

Passenger pick-up and drop-off (oKiss and Ride) could be facilitated on the upper (track) level from Duke street, providing direct access to the rail platforms

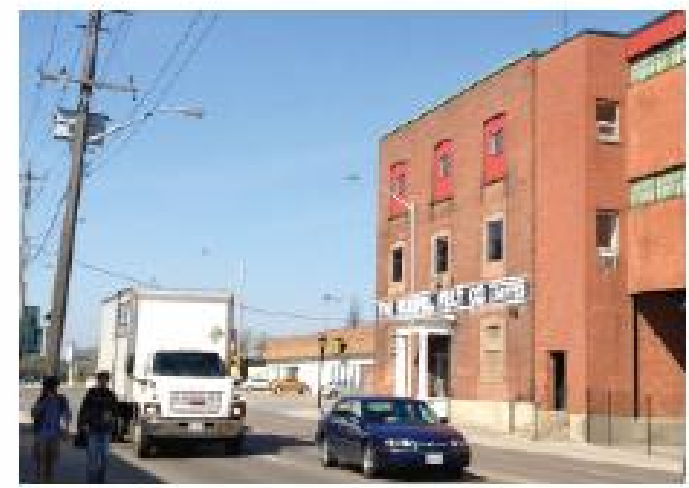

The original Rumpel Felt Building has cultural

8 heritage value and is intended to be adaptively reused as part of the Transit Hub plan. 


\subsubsection{Examining the extracting Concepts with the Three Dimensions}

Table1 the Appraisal of the Referential Set in King Victoria Hub Street Source: Researcher

\begin{tabular}{|c|c|c|c|c|c|c|c|c|c|c|c|c|}
\hline \multicolumn{13}{|c|}{ Concepts of Sustainable Commercial Streets } \\
\hline Dim. & Place Identity & & $\begin{array}{c}\text { Mixed } \\
\text { Uses }\end{array}$ & & $\begin{array}{c}\text { Pedestrian } \\
\text { Environment }\end{array}$ & & Accessibility & & Safety & & $\begin{array}{c}\text { Environmenta } \\
\text { aspects }\end{array}$ & \\
\hline \multirow{6}{*}{ 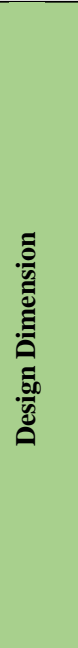 } & $\begin{array}{l}\text { Image and } \\
\text { Identity }\end{array}$ & G & $\begin{array}{l}\text { Mixed-use } \\
\text { development }\end{array}$ & G & $\begin{array}{l}\text { Comfortable } \\
\text { Movement }\end{array}$ & G & $\begin{array}{c}\text { Avoid Car } \\
\text { Oriented Plan } \\
\text { Design }\end{array}$ & $\mathrm{F}$ & $\begin{array}{c}\text { Dynamic } \\
\text { Streets }\end{array}$ & G & $\begin{array}{l}\text { Street and } \\
\text { Building } \\
\text { Orientation }\end{array}$ & $\mathrm{F}$ \\
\hline & $\begin{array}{l}\text { Symbolic } \\
\text { Meaning }\end{array}$ & G & $\begin{array}{l}\text { Avoid car- } \\
\text { Oriented Plan }\end{array}$ & $\mathrm{F}$ & $\begin{array}{l}\text { Clarify sidewalk } \\
\text { and crosswalk } \\
\text { configurations }\end{array}$ & $\mathrm{F}$ & $\begin{array}{l}\text { Connectivity and } \\
\text { permeable } \\
\text { space }\end{array}$ & G & $\begin{array}{c}\text { Safe } \\
\text { Sidewalks }\end{array}$ & G & $\begin{array}{l}\text { Minimize the } \\
\text { Direct Sun } \\
\text { Light }\end{array}$ & $\mathrm{G}$ \\
\hline & $\begin{array}{c}\text { Historic } \\
\text { districts streets }\end{array}$ & $\mathrm{F}$ & Mixed Land use & G & $\begin{array}{c}\text { Roadway and } \\
\text { engineering } \\
\text { measures, }\end{array}$ & $\mathrm{F}$ & $\begin{array}{c}\text { Pedestrians } \\
\text { Mobility }\end{array}$ & G & $\begin{array}{c}\text { Shelter and } \\
\text { security } \\
\text { Contact }\end{array}$ & G & $\begin{array}{c}\text { Albedo side } \\
\text { walk Design } \\
\text { pavements }\end{array}$ & $\mathrm{G}$ \\
\hline & $\begin{array}{l}\text { heritage } \\
\text { significance }\end{array}$ & $\mathrm{F}$ & & & $\begin{array}{l}\text { Programs to } \\
\text { enforce existing } \\
\text { traffic laws }\end{array}$ & $\mathrm{P}$ & Sidewalks Role & $\mathrm{G}$ & & & $\begin{array}{c}\text { Reduce the } \\
\text { Urban heat } \\
\text { island effect }\end{array}$ & $\mathrm{F}$ \\
\hline & & & & & $\begin{array}{c}\text { Forgiving vehicle } \\
\text { designs that } \\
\text { minimize } \\
\text { pedestrian injury }\end{array}$ & $\mathrm{F}$ & Directional Signs & G & & & & \\
\hline & & & & & & & Sidewalks Width & G & & & & \\
\hline \multirow{3}{*}{ 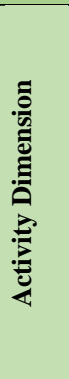 } & $\begin{array}{c}\text { Historical } \\
\text { dimensions }\end{array}$ & $\mathrm{F}$ & $\begin{array}{l}\text { location of } \\
\text { different } \\
\text { facilities }\end{array}$ & G & $\begin{array}{c}\text { Enhance the } \\
\text { Social Interaction }\end{array}$ & G & $\begin{array}{c}\text { More Dynamic } \\
\text { Space }\end{array}$ & G & $\begin{array}{c}\text { Physiological } \\
\& \text { feeling } \\
\text { needs }\end{array}$ & G & Mitigation & $\mathrm{F}$ \\
\hline & $\begin{array}{c}\text { Monumental } \\
\text { memory }\end{array}$ & $\mathrm{P}$ & $\begin{array}{l}\text { Various mode of } \\
\text { transportation }\end{array}$ & G & $\begin{array}{c}\text { visual traffic } \\
\text { calming }\end{array}$ & G & $\begin{array}{l}\text { Welcoming } \\
\text { Space }\end{array}$ & G & $\begin{array}{l}\text { Eyes on } \\
\text { Streets }\end{array}$ & G & Adaptation & $\mathrm{F}$ \\
\hline & & & $\begin{array}{l}\text { short amount of } \\
\text { time with low- } \\
\text { price }\end{array}$ & G & $\begin{array}{l}\text { Pedestrian } \\
\text { Environment } \\
\text { Control }\end{array}$ & G & Magnetic Nodes & G & $\begin{array}{l}\text { Pedestrian } \\
\text { Sociability }\end{array}$ & G & & \\
\hline \multirow{5}{*}{ 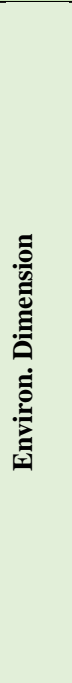 } & Preserving & $\mathrm{F}$ & $\begin{array}{c}\text { Short Travel } \\
\text { Distance }\end{array}$ & G & encourage walking & G & $\begin{array}{l}\text { Place Location \& } \\
\text { Surrounding }\end{array}$ & G & $\begin{array}{c}\text { Natural } \\
\text { Daylight }\end{array}$ & G & $\begin{array}{l}\text { Material } \\
\text { selection. }\end{array}$ & $\mathrm{G}$ \\
\hline & & & Local Retail & $\mathrm{F}$ & $\begin{array}{c}\text { Facilitate children } \\
\text { and handicapped } \\
\text { zones \& }\end{array}$ & $\mathrm{F}$ & $\begin{array}{c}\text { Building } \\
\text { Proportions and } \\
\text { Orientations }\end{array}$ & $\mathrm{F}$ & & & $\begin{array}{c}\text { Improve } \\
\text { rainwater } \\
\text { reuse quality. }\end{array}$ & $\mathrm{F}$ \\
\hline & & & & & $\begin{array}{c}\text { Pedestrians } \\
\text { Shading Devices }\end{array}$ & G & & & & & $\begin{array}{c}\text { Reduce } \\
\text { Environment } \\
\text { al Impacts }\end{array}$ & $\mathrm{G}$ \\
\hline & & & & & & & & & & & $\begin{array}{c}\text { Reduce } \\
\text { energy } \\
\text { consumption }\end{array}$ & $\mathrm{F}$ \\
\hline & & & & & & & & & & & $\begin{array}{c}\text { Waste } \\
\text { Management } \\
\text { and } \\
\text { Recycling }\end{array}$ & $\mathrm{G}$ \\
\hline
\end{tabular}

Key Table $\quad$ Good $=\mathrm{G} \quad$ Fair $=F \quad$ Poor $=\mathrm{P}$ 


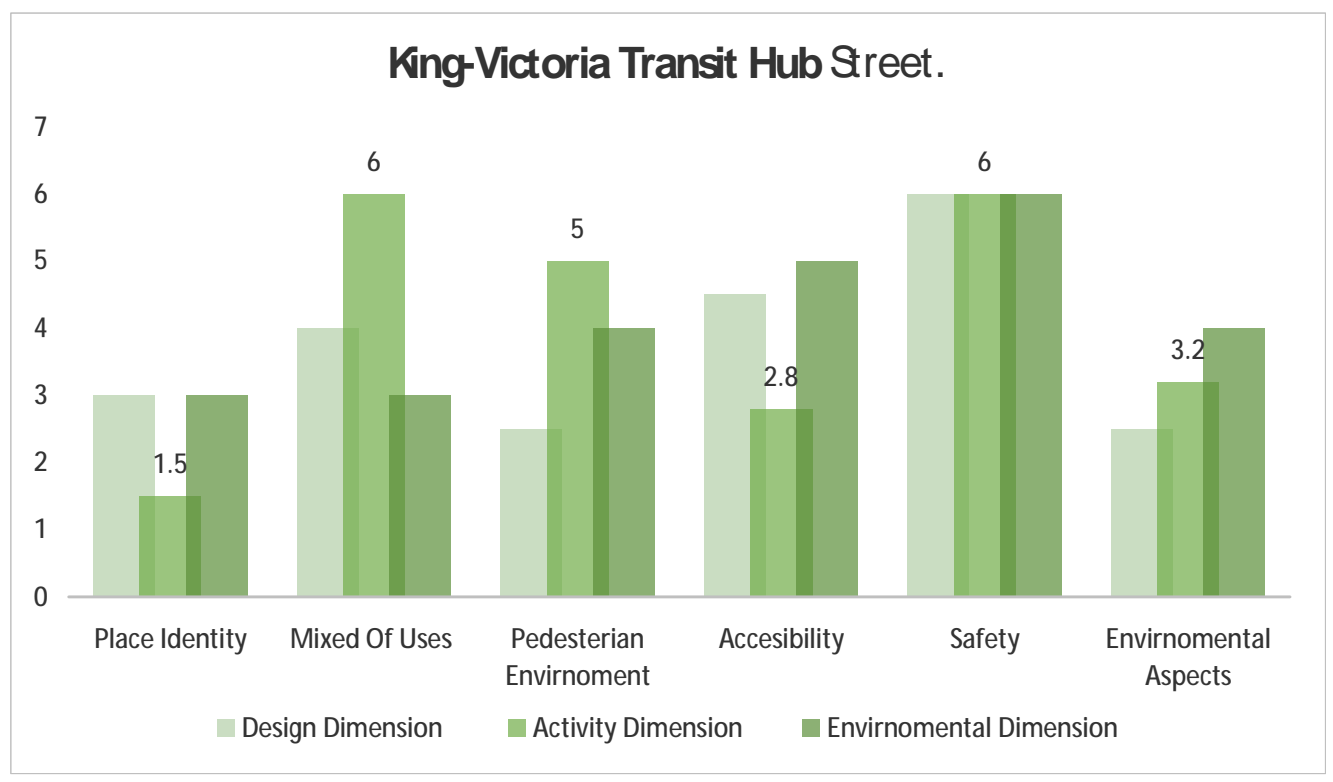

Figure Chart Shows the Percentage Of sustainable Target in the Street source: The Researcher

\subsection{Second Examine Case: Al Gouna Down Town Commercial Street.}

ALGouna Downtown Commercial Public Space area; where most of the commercial facilities are found. Which is mainly a pedestrian square full of outdoor cafes and Open restaurants, developed and owned by Orascom Hotels and Development (OHD), da ng from about 1990 in the Gouna Village.

The Space planning and design is compact and is characterized by the visibility of its entrance. It has been taken into consideration that the character of the city development is derived from the traditional rural Egyptian context Buildings are covered by domes and vaults and streets with Porous paving materials or a bright asphalt in order to provide the interior with moderate climate, (Nady,R. 2008).

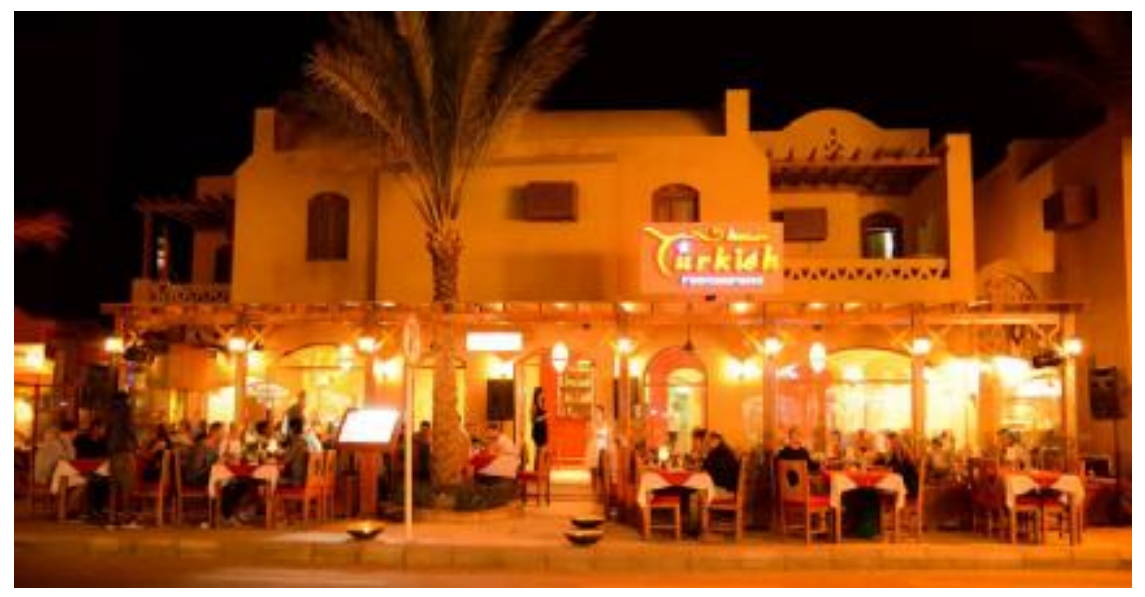

Figure : Down town cafeterias Source: Algouna state of mind magazine 

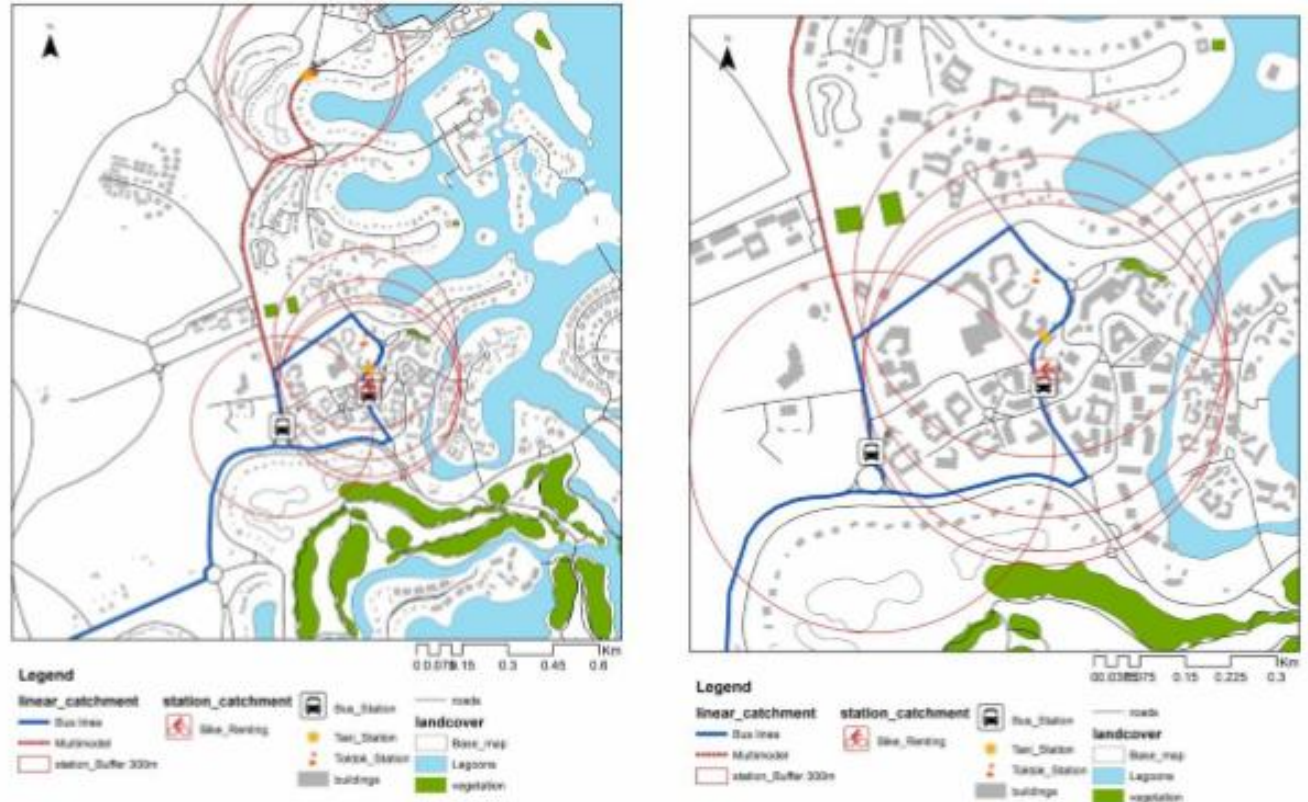

Figure 3 Accessibility map for AlGouna source: Ahmed Khalil

\subsubsection{Examining the extracting Concepts with the Three Dimensions}

Table: Shows the Appraisal of the Referential Set in King Victoria Hub Street Source: Researcher

\begin{tabular}{|c|c|c|c|c|c|c|c|c|c|c|c|c|}
\hline \multicolumn{13}{|c|}{ Concepts of Sustainable Commercial Streets } \\
\hline Dim & $\begin{array}{c}\text { Place } \\
\text { Identity }\end{array}$ & & $\begin{array}{l}\text { Mixed } \\
\text { Uses }\end{array}$ & & $\begin{array}{l}\text { Pedestrian } \\
\text { Environment }\end{array}$ & & ccessibility & & Safety & & $\begin{array}{l}\text { nvironment } \\
\text { aspects }\end{array}$ & \\
\hline \multirow{6}{*}{ 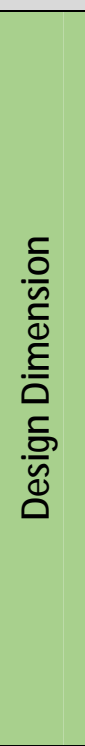 } & $\begin{array}{l}\text { Image and } \\
\text { Identity }\end{array}$ & G & $\begin{array}{c}\text { Mixed-use } \\
\text { development }\end{array}$ & G & $\begin{array}{c}\text { Comfortable } \\
\text { Movement }\end{array}$ & G & $\begin{array}{l}\text { Avoid Car } \\
\text { Oriented Plan } \\
\text { Design }\end{array}$ & $\mathrm{F}$ & $\begin{array}{l}\text { Dynamic } \\
\text { Streets }\end{array}$ & G & $\begin{array}{c}\text { Street and } \\
\text { Building } \\
\text { Orientation }\end{array}$ & $P$ \\
\hline & $\begin{array}{l}\text { Symbolic } \\
\text { Meaning }\end{array}$ & G & $\begin{array}{l}\text { Avoid car- } \\
\text { Oriented Plan }\end{array}$ & $\mathrm{F}$ & $\begin{array}{l}\text { Clarify sidewalk } \\
\text { and crosswalk } \\
\text { configurations }\end{array}$ & G & $\begin{array}{l}\text { Connectivity } \\
\text { and permeable } \\
\text { space }\end{array}$ & G & $\begin{array}{c}\text { Safe } \\
\text { Sidewalks }\end{array}$ & G & $\begin{array}{l}\text { Minimize } \\
\text { the Direct } \\
\text { Sun Light }\end{array}$ & $\mathrm{F}$ \\
\hline & $\begin{array}{l}\text { Historic } \\
\text { districts } \\
\text { streets }\end{array}$ & $\mathrm{p}$ & M ixed Land use & G & $\begin{array}{l}\text { Roadway and } \\
\text { engineering } \\
\text { measures, }\end{array}$ & $\mathrm{F}$ & $\begin{array}{c}\text { Pedestrians } \\
\text { Mobility }\end{array}$ & G & $\begin{array}{l}\text { Shelter and } \\
\text { security } \\
\text { Contact }\end{array}$ & G & $\begin{array}{c}\text { Albedo side } \\
\text { walk Design } \\
\text { pavements }\end{array}$ & $P$ \\
\hline & $\begin{array}{l}\text { heritage } \\
\text { significance }\end{array}$ & $p$ & & & $\begin{array}{l}\text { Programs to } \\
\text { enforce existing } \\
\text { traffic laws }\end{array}$ & $\mathrm{F}$ & Sidewalks Role & G & & & $\begin{array}{c}\text { Reduce the } \\
\text { Urban heat } \\
\text { island effect }\end{array}$ & $P$ \\
\hline & & & & & $\begin{array}{l}\text { Forgiving vehicle } \\
\text { designs that } \\
\text { minimize } \\
\text { pedestrian injury }\end{array}$ & $\mathrm{F}$ & $\begin{array}{l}\text { Directional } \\
\text { Signs }\end{array}$ & G & & & & \\
\hline & & & & & & & $\begin{array}{l}\text { Sidewalks } \\
\text { Width }\end{array}$ & $\mathrm{F}$ & & & & \\
\hline \multirow{3}{*}{ 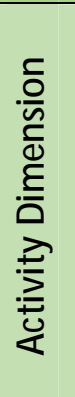 } & $\begin{array}{c}\text { Historical } \\
\text { dimensions }\end{array}$ & $p$ & $\begin{array}{l}\text { location of } \\
\text { different } \\
\text { facilities }\end{array}$ & G & $\begin{array}{l}\text { Enhance the } \\
\text { Social } \\
\text { Interaction }\end{array}$ & $\mathrm{F}$ & $\begin{array}{l}\text { More Dynamic } \\
\text { Space }\end{array}$ & G & $\begin{array}{l}\text { Physiologica } \\
\text { I \& feeling } \\
\text { needs }\end{array}$ & G & Mitigation & $\mathrm{F}$ \\
\hline & $\begin{array}{l}\text { Monument } \\
\text { al memory }\end{array}$ & $P$ & $\begin{array}{c}\text { Various mode } \\
\text { of } \\
\text { transportation }\end{array}$ & G & $\begin{array}{l}\text { visual traffic } \\
\text { calming }\end{array}$ & $\mathrm{F}$ & $\begin{array}{l}\text { Welcoming } \\
\text { Space }\end{array}$ & G & $\begin{array}{l}\text { Eyes on } \\
\text { Streets }\end{array}$ & G & Adaptation & $\mathrm{F}$ \\
\hline & & & $\begin{array}{l}\text { short amount } \\
\text { of time with } \\
\text { low-price }\end{array}$ & $\mathrm{F}$ & $\begin{array}{l}\text { Pedestrian } \\
\text { Environment } \\
\text { Control }\end{array}$ & G & $\begin{array}{l}\text { Magnetic } \\
\text { Nodes }\end{array}$ & G & $\begin{array}{l}\text { Pedestrian } \\
\text { Sociability }\end{array}$ & G & & \\
\hline
\end{tabular}




\begin{tabular}{|c|c|c|c|c|c|c|c|c|c|c|c|c|}
\hline \multirow{5}{*}{ 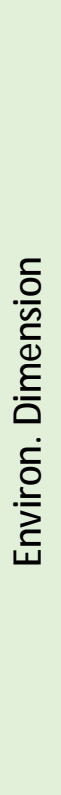 } & Preserving & $F$ & $\begin{array}{c}\text { Short Travel } \\
\text { Distance }\end{array}$ & G & $\begin{array}{l}\text { encourage } \\
\text { walking }\end{array}$ & G & $\begin{array}{l}\text { Place Location } \\
\& \text { Surrounding }\end{array}$ & G & $\begin{array}{l}\text { Natural } \\
\text { Daylight }\end{array}$ & G & $\begin{array}{l}\text { Material } \\
\text { selection. }\end{array}$ & G \\
\hline & & & Local Retail & G & $\begin{array}{c}\text { Facilitate } \\
\text { children and } \\
\text { handicapped } \\
\text { zones \& }\end{array}$ & $F$ & $\begin{array}{c}\text { Building } \\
\text { Proportions } \\
\text { and } \\
\text { Orientations }\end{array}$ & G & & & $\begin{array}{c}\text { Improve } \\
\text { rainwater } \\
\text { reuse } \\
\text { quality. }\end{array}$ & $F$ \\
\hline & & & & & $\begin{array}{c}\text { Pedestrians } \\
\text { Shading Devices }\end{array}$ & G & & & & & $\begin{array}{c}\text { Reduce } \\
\text { Environmen } \\
\text { tal Impacts }\end{array}$ & $F$ \\
\hline & & & & & & & & & & & $\begin{array}{c}\text { Reduce } \\
\text { energy } \\
\text { consumptio } \\
n\end{array}$ & G \\
\hline & & & & & & & & & & & $\begin{array}{c}\text { Waste } \\
\text { Managemen } \\
\mathrm{t} \text { and } \\
\text { Recycling }\end{array}$ & G \\
\hline
\end{tabular}

$\begin{array}{llll}\text { Key Table } & \text { Good }=\mathrm{G} & \text { Fair }=\mathrm{F} & \text { Poor }=\end{array}$

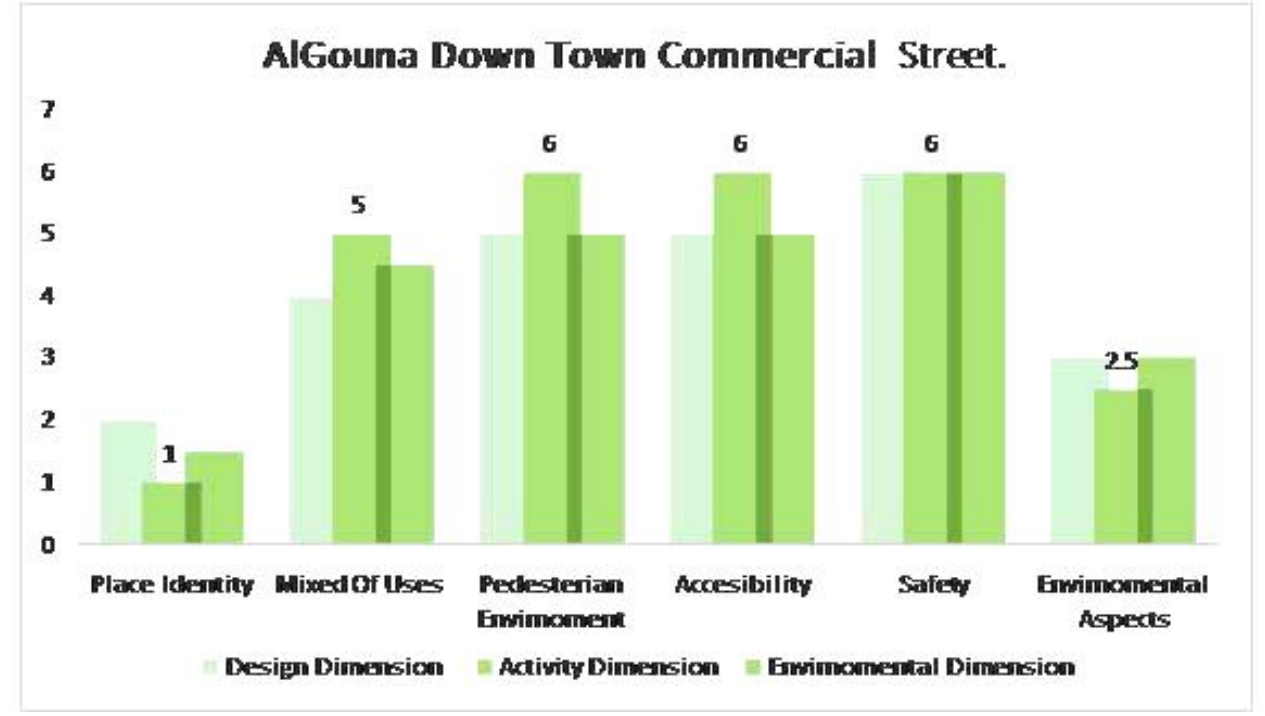

\section{Results Discussion:}

Figure: Chart Shows the Percentage Of sustainable Target in the Street source: The Researcher

\subsection{King Victoria Results and Discussion:}

\section{KINA G-YCTORLA ITRANMT HUR STREET.}

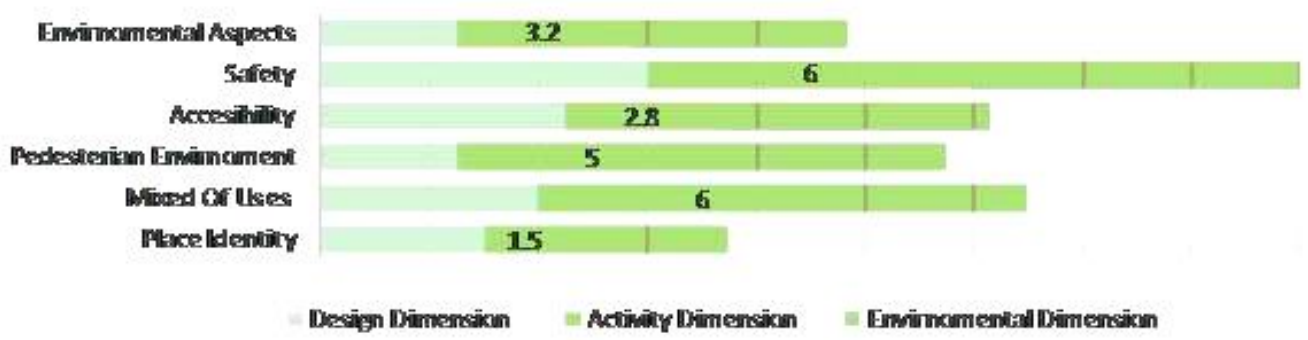

Figure: Chart Shows the Percentage Of sustainable Target in the Street source: Researcher 
From the evaluation Results in King Victoria transit hub Street, the sustainable achievement is quiet fine as the six concepts are presented by a constant rate , as the "Mixed uses " is presented by more than an activity in this active travelling area with various method of transportation in the street which encourage more people and more activity to be held in the street , while the "safety " method is also fine as the concept of" eyes on streets" were been applied through the presence of passerby and shop owners most of the time which give the street a self-safety use. In the other hand the place identity where been naturally present as the historical train station is located in the street. While the "Accessibility" and "pedestrian Environment "where been facilitated during the design development and provide a special underground bridge for the travelers and pedestrian from the train station to all the other street nodes with a clear and well-lighted sidewalk for all the users. What is worth to mention that the previous 5 dimensions are represented by a fine percent but the Environmental aspects are the lowest evaluations in the street as they concentrated only on the recycling methods but ignored any way for using a water management system that can be very useful especially in this zone of the street especially in winter.

\subsection{Algouna Down Town Street Results and Discussion:}

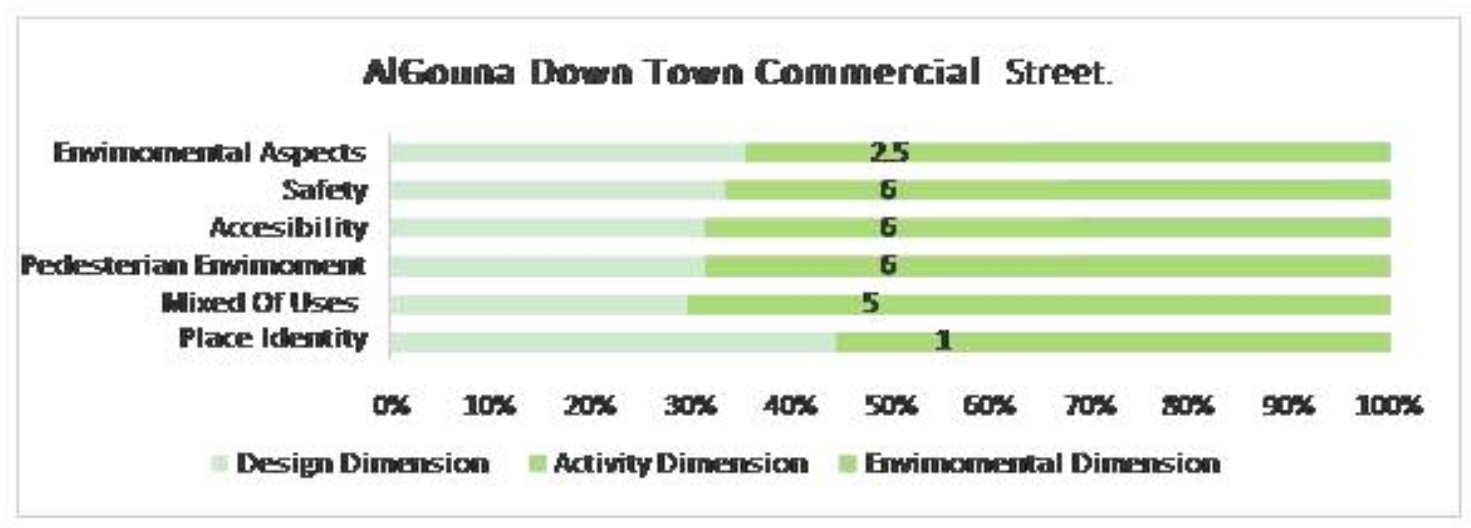

Figure: Chart Shows the Percentage Of sustainable Target in the Street source: The Researcher

In Al Gouna Down Town Street Evaluation Case the state of sustainability is presented in the Six Concepts as shown in the previous figure with a high pedestrian, safety and Comfortable Movement with accessible methods for the street as the toktok where all the users can feel comfort and surrounded by a well-environmental methods and ways to enhance new ways and sustain-durable materials that are used either in buildings and pavements in the street.

Moving to the various activities in the area for shopping and sitting and the street festivals every Sunday which give the street an attractive node for the pedestrians that come to al Downtown Street. The depending of the renewable energy in many fields of the street as lighting and waste management's make the environmental aspects present with moderated Rate

The Defects were clear as the historical identity is not clear as the place is a new place but they are moving on creating a well space identity through the dessert architecture of domes and vaultes to be stored as a different image for this street and to be remembered for even if it's not an origin from AlGouna City 


\section{CONCLUSION}

Regardless of That the Two Studied Examples are in different in context and regions but the referential set got through their strength and weak indicators, therefore the conclusion can be illustrated those Points.

First the six Concepts are found in the Two Different Examples, so this point shows how much the link and the relation between those concepts and the State of sustainability in current sustainable streets and the Concepts Indicators of the referential Set.

Another Conclusion was noticed that although the six concepts were shown in the successful Commercial Streets but in the "Environmental Aspects" are not focused on and a Lack of Environmental Dimension Applications in the Streets, that does not take the importance it should take to keep the Commercial Streets Green and sustainable within the Nature Integration and Enhancing of renewable Resources Technologies.

The Research considered these six concepts as the Concepts of Sustainable Commercial Streets. As they all imperative to reach the state of sustainability, they are: place identity, Mixed of Uses, Pedestrian Environment, Accessibility, Safety and The Environmental Aspects. Coordinating each concept's indicator in design, activity and Environmental Dimensions shaped the Reference table. This table is set into two axials. It consists horizontally from the six concepts and vertically from the three dimensions.

The Referential set was tested on two successful examples, King Victoria and Al gouna Down Town Streets. The six concepts were found together in the two examples; however, they are different contexts and morphology and their cycle of sustainability is different from each example. This confirms and approved the important of those 6 concepts together to reach the sustainable commercial street Target. The two examples achieved minimum of $85 \%$ for the referential set indicators.

\section{REFERENCES}

1. Al-Hagla, Khalid, (2004), Medina Transformation versus Sustaining Identity: Vitality of Medina Genetic Characteristics towards Cultural Sustainability, Alexandria, Egypt: Case Study, Paper presented in: International Seminar "The Mediterranean Medina “"

2. Universita' “G. D’Annunzio" - Facoltà di Architettura. Pescara, Italy

3. Al-Hagla, Khalid, (2005), Sustaining Cultural Identity of the Mediterranean Basin, APJ' Architecture and Planning Journal, Issue No. 16. - Faculty of Architectural Engineering, Beirut Arab University, Beirut, LEBANON.

4. Alexander, C. (2001, 2002, 2005). The Nature of Order: Books 1, 2, and 3, Center for Environmental Structure, Berkeley, California, USA

5. Ahern, J. (1995). Greenways as a Planning Strategy, Landscape and Urban Planning, Vol. 33.

6. Anon., (1997). Measuring Accessibility: An Exploration of issues and Alternatives. Environment and planning, volume A 29, p.75-94

7. Anon., (2012). Pedesteriazation Of Commercial Streets. In: Reclaiming Public Space A Situation Analysis of Commercial Street in Bangalore. India: Evangelical Social Action Forum.

8. Balsas, C.J.K. (2004). Measuring the Livability of an urban Center: an exploratory Study of key Performance Indicators, planning, Practice and Research,pp.101-110

9. Ciolek. (1978). Spatial Behaviour in Pedesterian Areas Ekistics, pp.120-122

10. Ching. (1992). Ordering Principels. In: London, ed. Architecture, form, space and Order Book. s.1.:3rd edition, pp. 339-340.

11. Council, A. D. P. (2006). Abu Dhabi Planning Council 2050. First edition ed. Abudhabi: UAE PLanning council. 
12. Council, A. P., (2004). American Planning Association. In: Chicago: s.n., pp. 245248.

13. Hannallah,Gihan Mosaad R. N. F. (2014). Zero-Carbon Cities as a New Realization of Sustainable Cities. BUE-FISC, p. 124.

14. Government, t. s. (2012). A Myth of Urban Design: The 'Sense of Enclosure' Theory.

15. Jacobs. (1993). Great Streets. s.l.:Cambridge : MA: The MIIT Press..

16. Jacobs, J. (1961). The Uses of Sidewalks. s.l.:s.n.

17. Jones, P. (2008). Creating more people-friendly urban. s.l., s.n.

18. Kaplan, R.K.S. (1989). Environment Preference: A comparison of Four Domains of predictors. Environment and Behavior, pp.21.509-530

19. Kendall, S. (2002). “An Open Building Strategy for Balancing Production Efficiency and Consumer Choice in Housing". Proceedings (vol 1 of 2) 10th Annual Symposium Construction Innovation and Global Competitiveness. University of Cincinnati.Ohio. 


\title{
TOWARDS SUSTAINABLE COMMERCIAL STREETS
}

\author{
Marwan Ali Fouad, \\ Institute of Graduate Studies and Research, Alexandria University, Alexandria, Egypt \\ Marwan@apgarche.ae
}

\begin{abstract}
The public space has always been the focus for many researchers, especially the controlling of the space through the urban configurations. The street space experience has been introduced as an approach to rethink in the public areas with a new method of thinking and how it affects the durability of streets to become sustainable within various dimensions of analytical studies. This Paper aims to set an approach for measuring and evaluate the state of Sustainability in the streets and public spaces through the previous studies done and extract six-different concepts within the three Sustainable dimensions that affect the street users which are (design configurations, activity and Environmental aspects). Moreover these criteria can highlight the commercial street problems in the Egyptian Streets and determine the strong and weakness points through creating an Evaluation Reference set through harmonizing the concepts with the Dimension approach and apply this reference set on the streets for measuring state of sustainability. All this result would help to set recommendations for better public spaces and protect the city character, enhance the streets that characterize the city, neglecting all the random unorganized activities that affect directly the pedestrians, and finally enhancing the controlling of the sustainable public space. on the other hand the paper also works to find a set of scientific recommendations for planning and the need to take into account during the process of urban design with the integration of the environment, a basic standard to be based on environmental and design criteria without affecting the resources and needs of future generations.
\end{abstract}

Keywords: Commercial streets, sustainable streets, Environmental Aspects, Street activity, Accessibility, Pedestrians, Place identity.

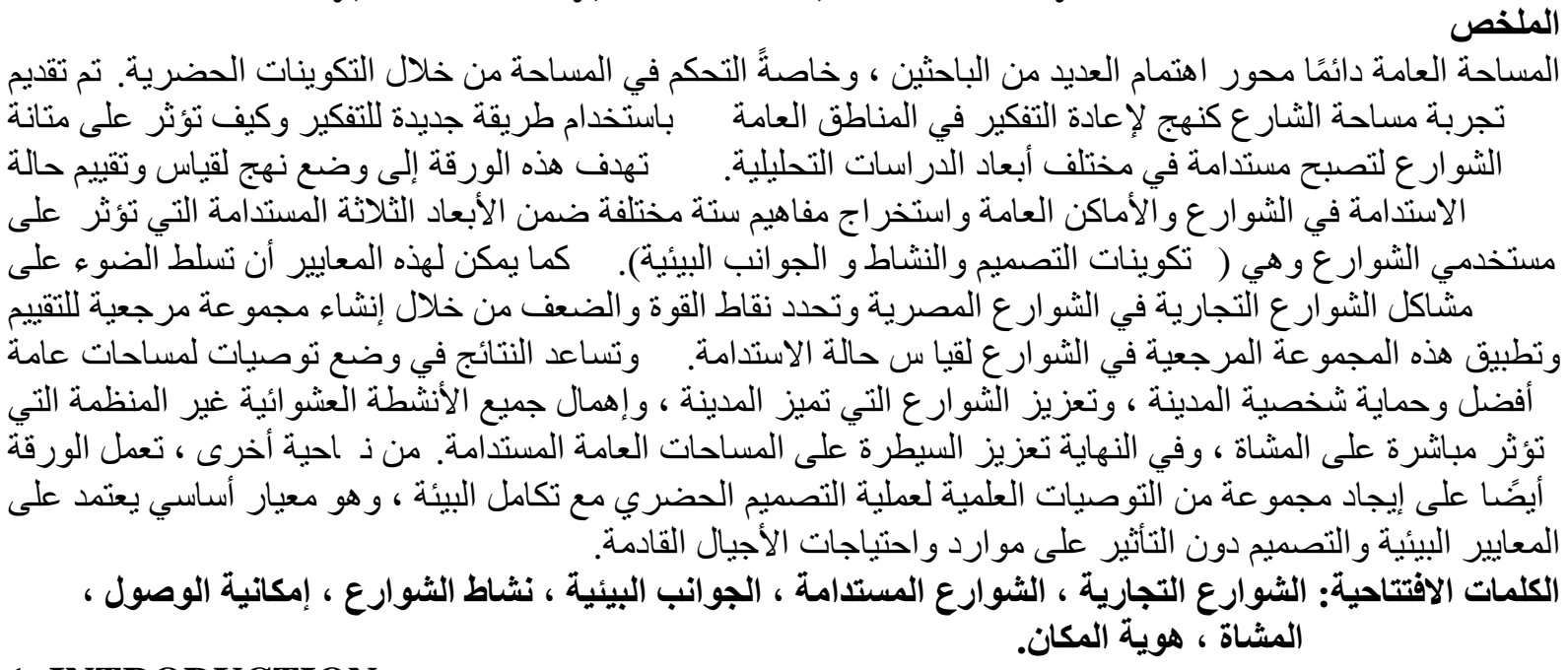

\section{INTRODUCTION}

The public space is changing. Pedestrians are now living in a world with a negative environment, streets radically different. These new spaces vary in quality and intensity from 
those of the past; however, many researchers have been alerted to the dangers of the random spread of loss in control that lead to informal streets into every Public Space.

Every Public space has a spatial character which affected by proportion, scale, size of parts relative to each other, color, texture, furnishing and finally its activities. We need to develop an appropriate understanding of them and to treat them as dynamic physical objects with a multi-dimensional element, (Gontier, 2006).More Over the public space must link the main public urban space pillars which are the Life activities of the users in the space then the Space place which took place within the area and the surrounding Buildings that will enclose the space around it to generate the whole Public Space Image (Gontier, 2006).

A Street that is always alive with people and movement is an important and significant street, rather than a dead one where no one passes or not used frequently. Moreover, the more the street becomes full of people, the more people feel safer with the presence of the others. The job of handling strangers mostly depends on the streets of a city (J. Jacobs, 1961).

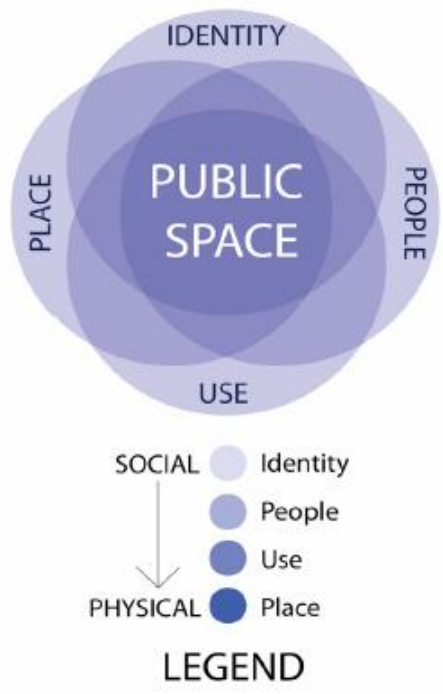

Figure 1: Public Space Characteristics Source: Brcelona Council 2004

Commercial streets are the Areas in the city that intended to provide a variety of Shopping, activities, and service options and convenience. (Planning council, 2012) The commercial streets primarily c0mposed of commercial building or shops Where the Commercial activity took place as the a downtown, central business district, financial district, "Main Street", commercial strip, or shopping center includes the buying and selling of goods and services in retail businesses, While commercial activities typically take up a relatively small amount of land, that are enhancing the community economy.

According to (JONES, 2008) Urban commercial streets provide the setting for a wide range of urban street activities, which can be grouped under two street functions of 'Place' and 'Accessible Link'.

As an Accessible Link, a street provides an accessible tube for movement, and forms an integral part of the wider urban street network and other, more specialized, urban transport networks, A Link user may travel by a variety of modes, from private car or truck to bus, bicycle or on foot. Their essential need is to follow a continuous, linear path through the street network, with minimum disruption and a seamless connection from the beginning to the end of their journey. In general, they are seeking to minimize travel time along each section of street.

As a Place, Urban Commercial Street is a destination in its own right: a location where activities occur on or adjacent to the street. A Place user is someone wishing to make use of some of the features that are on that particular street, and will usually do so on foot. While such people are classified as 'pedestrians', they are not passing through the area - they are spending time in the area, and may be carrying out a wide variety of activities (e.g. shopping, 
talking, waiting, resting, working). They are particularly affected by the noise and air pollution produced by vehicular traffic, and the general severance effect of heavy traffic volumes in inhibiting their movement between places on opposite sides of the street. However, not all of the traffic and transport-related activity observed on urban streets is part of that street.

\section{Sustainable Streets Strategy}

In order to convert the Traditional Negative Streets into an effective positive Streets with activities and Design a place that can serve the current generations without affecting the new generations it should be developed on a Sustainable strategy way which can be named as a sustainable Development Plan but before that we should Review the sustainability and Its Main Pillars that affect the Urban Space.

A generally accepted definition of sustainable development, and a good point to begin an exploration of this concept, is taken from the Brundtland Report: 'Sustainable development is development that meets the needs of the present generation without compromising the ability of future generations to meet their own needs' (World Commission on Environment and Development, 1987).

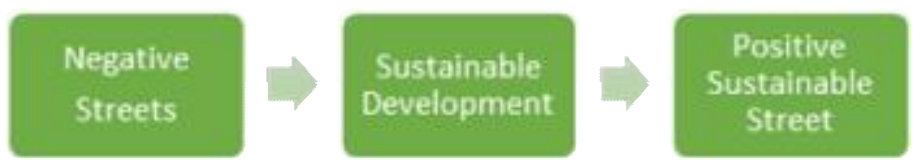

Figure 2: Sustainable Street Strategy Through Sustainable Development Source: Author.

All the Previous Studies that studied the Sustainable Streets in the field of Urban Design and Sustainability Basic Pillars linked them to certain Concepts that are important and necessary for judgment and results. In achieving the sustainable streets and control them several theorists literally linked Sustainable streets to certain concepts (Lukes, 2008) (Anon., 2006) (Jacobs, 1993) (Lynch, 1960) and according to all those researchers negotiate the management strategy for the Commercial Streets that can be listed in the below Points:

- Make a unique local identity and civic pride

- Shape the existing and future development and

- Enhance the physical character of an area,

- Improve the social integration, including accessibility

- Provide connected routes between Street nodes recreation, walking and cycling, and safer routes Schools.

- Protect and enhance the biodiversity.

- Re-provide green infrastructure and ecosystem Services.

- Increase and boost the potential of shopping, which will increase the economical income. and Cultural activities provide cultural, social, recreational, sporting and community facilities

- Promote understanding of the historical, cultural and archaeological value of place identity.

- Contribute to the create a safe and healthy place, Including quiet areas

- Provide popular outdoor commercial facilities

- Help mitigate and adapt to climate change

- Improve environmental opportunities to enjoy contact with the natural world.

This illustrates the Necessity of the Presence of all those studies and Concepts in order to understand the Sustainable Streets. A referential Set must be exported from a big Number of literature and theoretical Previous Studies in order to deduce this set of Concepts.

Six Main Concepts were extracted from theoretical Reference, Each Concept was studied by theorists in order to gain indicators that could be used to recognize the presence of such a concept. 
Table 1: Main Theoretical Concept extracted from Previous Studies source: Developed by Author.

\begin{tabular}{|c|c|c|}
\hline Concept & Example & $\begin{array}{l}\text { Theoretical } \\
\text { Reference }\end{array}$ \\
\hline A. Place Identity & $\begin{array}{l}\text { "Iconic Streets Imbued with a lasting Image and Grounded } \\
\text { sense of place is Essential Component to all Urban and rural } \\
\text { Cities. Not all streets can have a city-wide Iconic Identify. } \\
\text { However, some Streets take on that role of telling the story of the } \\
\text { area and the larger region it represents. " }\end{array}$ & $\begin{array}{l}\text { (Anon., 2006) } \\
\text { (Hague, 2005) } \\
\text { (Jacobs, 1993) } \\
\text { (Harmouth 1990) } \\
\text { (Proshansky, 1978) }\end{array}$ \\
\hline $\begin{array}{l}\text { B. Mixed of Uses } \\
\text { (Activity) }\end{array}$ & $\begin{array}{l}\text { "Activities are the basic Building Blocks of any place. Having } \\
\text { something to do gives people a reason to come to a place -and } \\
\text { Return. when there is nothing to do, a space will be empty and } \\
\text { that generally Means that something is wrong " }\end{array}$ & $\begin{array}{l}\text { (Petersen 2006) } \\
\text { (Brtonetal ,2003) } \\
\text { (Gtz1999) } \\
\text { (Tibbalds ,1992) }\end{array}$ \\
\hline $\begin{array}{ll}\text { C. } & \begin{array}{l}\text { Pedestrian } \\
\text { Environment }\end{array}\end{array}$ & $\begin{array}{l}\text { "Pedestrian life streets are full of life that enhance pedestrian } \\
\text { safety and mobility for all the users of the street." }\end{array}$ & $\begin{array}{l}\text { (Elhamy, 2012) } \\
\text { (Evans, 2009) } \\
\text { (Senbel,2008) } \\
\text { (Bentley, 1985) } \\
\text { (Gehl ,1989) }\end{array}$ \\
\hline D. Accessibility & $\begin{array}{l}\text { "Building an active Places and path ways of Streets for People, } \\
\text { they Need them to be accessible. when they are not we are } \\
\text { excluding people from participating in the social or economic } \\
\text { activity that is going on in that space" }\end{array}$ & $\begin{array}{l}\text { (Lynch, 1960) } \\
\text { (Jacobs, 1961) } \\
\text { (Gehl ,1989) }\end{array}$ \\
\hline E. Safety & $\begin{array}{l}\text { "when People say that a city, or a part of it, is dangerous or is a } \\
\text { jungle what they mean primarily is that they do not feel safe on } \\
\text { the sidewalks" }\end{array}$ & $\begin{array}{l}\text { (Lyshan 2014) } \\
\text { (Evans, 2009) } \\
\text { (Jacobs, 1961) } \\
\text { (Mslow 1954) }\end{array}$ \\
\hline $\begin{array}{l}\text { F. Green } \\
\text { Environmental } \\
\text { Aspects }\end{array}$ & $\begin{array}{l}\text { "Effectively incorporating green techniques into the Street } \\
\text { network provides significant opportunity to decrease } \\
\text { infrastructure demands and pollutant transport and change the } \\
\text { street Life" }\end{array}$ & $\begin{array}{l}\text { (PoolR., 2009) } \\
\text { (Ryser J.2009) } \\
\text { United Nations -Human } \\
\text { Settlement 2009) }\end{array}$ \\
\hline
\end{tabular}

\section{DIMENSIONS OF SUSTAINABLE COMMERCIAL STREETS}

As a result, from the Previous Studies for the Existence of the Sustainability in the Urban Streets can be easily recognized when taking in consideration the three Sustainable Pillars (social. Economic and Environmental) Pillars with the previous Studies of (Jacobs, 1993) (Greenberg, 2009), (Lynch, 1960) (lehmann, 2010) and (Jones, 2008) in the concepts of successful green Street.

\section{Design Configurations Dimensions}

Create A network that supports Communities and places

Integrate the streets with the natural Different Scales systems

Respect the Current Natural Existing

Environment

Mixed Land use activity.

Pedestrian opportunity.

Variety of transportations. (Maximize

Transportations Facilities).

\section{Activity Dimensions}

Increase the social

Interaction.

Pedestrian Activity.

Welcoming interactivity.

Enhancing Security and safety.

Encouraging Community

Participation.

\section{Environmental Dimensions}

Reduce impacts on

environmental resources.

Reduce energy consumption.

Improve rainwater reuse quality.

Recycling Methods.

Material selection For Streets

and Pavements.

Figure 3: three sustainable public space dimensions Source: Developed by Author.

The Relation between the users of the street to the urban space can be affected by the Activity that take place in the street, also affected by the Design configurations that are managed and planned to fit all the user needs to give them a comfort safe place to make their activities within a well Environmental Zone surround all the street elements to reach the target of sustainability in the street space. 
Therefore the sustainable streets were linked in the theory with the six concepts: place identity mixed of uses, pedestrian environment, accessibility, safety and environmental aspects. These Concepts were Very Important for deducing the Sustainability Of every Street, the link was stated directly or in directly in the previous Studies. Each of the six Concepts Expressed and linked to the three concluded Sustainable Dimension: Design, Activity and Environmental Dimensions to extract the Criteria of the Sustainable Commercial Streets.

The six Concepts were found to have an important role in theory which requires their integration Together.

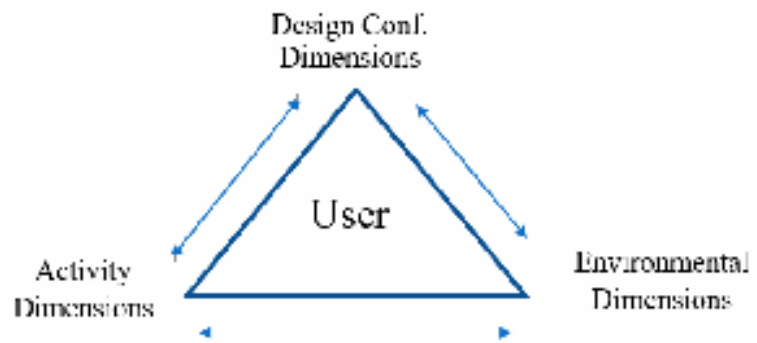

Figure 4: the relation between the street user and the Three Dimensions, source: Author.

\section{CONCEPT ANALYSIS METHODOLOGY}

The main methodology is to study each concept separately across the three dimensional pillars through the Previous Studies and generate the main elements for each concept to set the street actual sustainable elements.

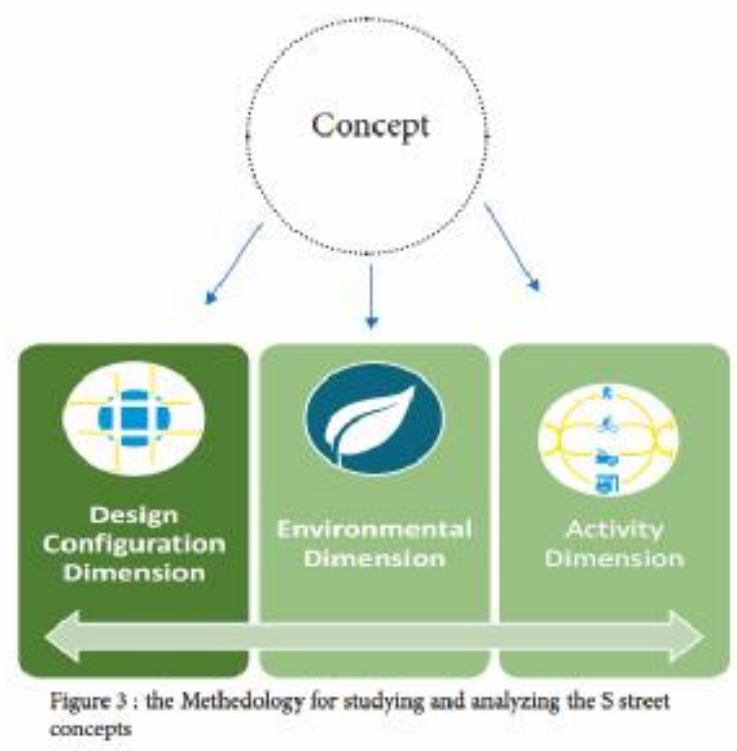

\subsection{Place Identity as a Concept:}

Lately, people have been talking increasingly about "loss of the place identity." What they're missing is the kind of place that has been special in their lives and given them a strong sense of community and well-known character for the surrounding Urban space elements that share a known unique character.

Iconic Streets Imbued with a lasting Image and Grounded sense of place is Essential Component to all Urban and rural Cities. Not all streets can have a city-wide Iconic Identify. However, some Streets take on that role of telling the story of the area and the larger region it represents. (Anon., 2006)

The Place identity or image of the place and in some ways it is related to the concepts of Place attachment and Sense of place. Refers to a cluster of ideas about place and identity in 
the fields of geography, urban planning, urban design, landscape architecture, environmental psychology and urban /ecological sociology. It concerns the meaning and significance of places for their inhabitants and users, and how these meanings contribute to individuals' conceptualizations of self. (Hague, 2005).

According to (Realm, 2016) image and identity street types can be supported and facilitated through the design process of the street environment and been divided according to the scale, facilities,

Activities and the street historical background, First Downtown main streets, as the original historic commercial mixed-use traffic street continue to be a major thoroughfare for the city, but also function as an important work center, public gathering space, administrative core and shopping destination. Clearly marked and signaled crosswalks and intersections, high quality furnishings and streetscape plantings.

Hormuth (1990) discusses the role of relocation and self-concept change suggesting that choosing to move can represent self-concept change with the old place becoming a symbol of the old self and the new place representing an opportunity to develop new identities. In both these examples place is considered to be an active part of the construction of a person's identity, representing continuity and change.

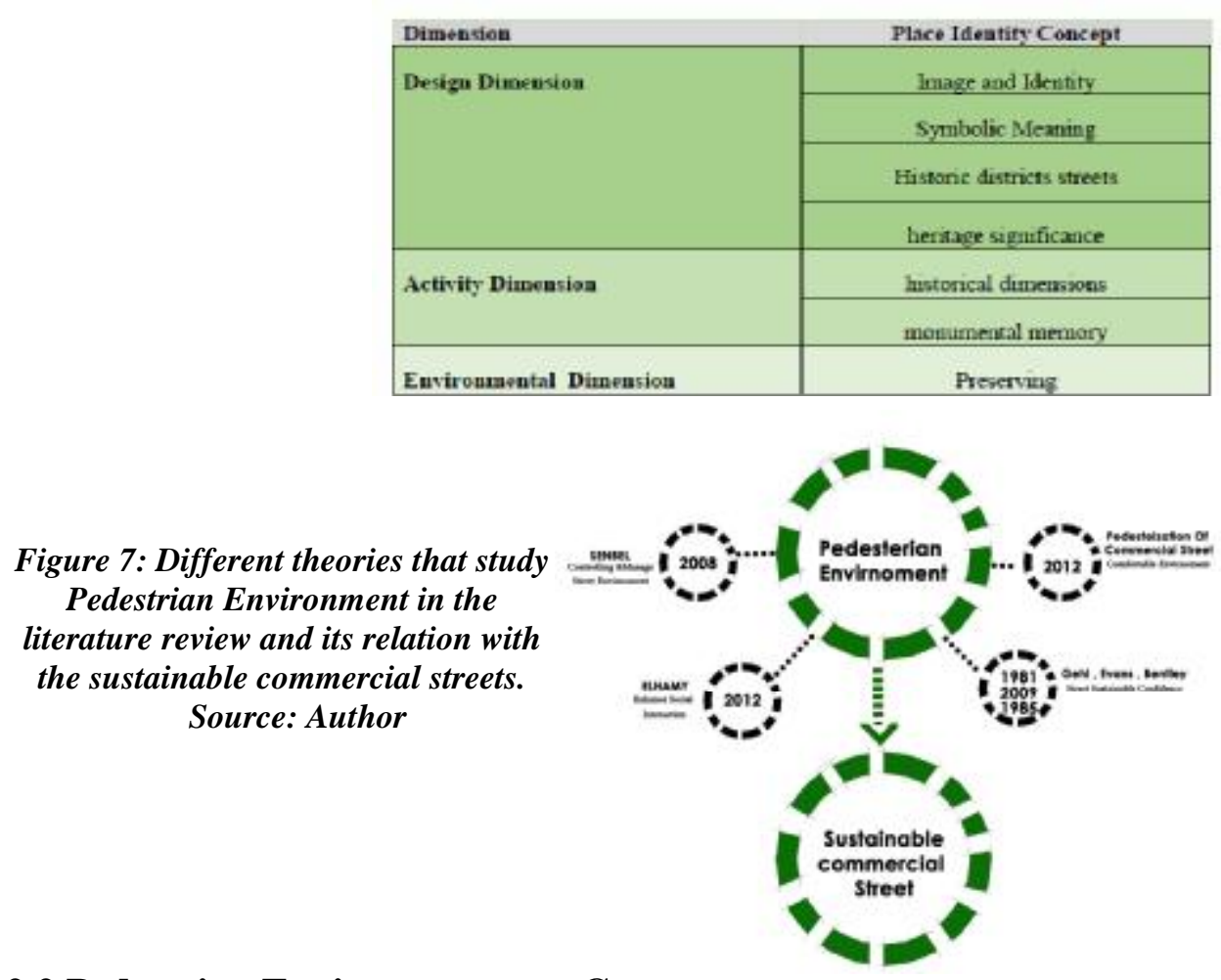

\subsection{Pedestrian Environment as a Concept}

Pedestrian environment (also known as auto-free zones and car-free zones) are zones of a city or town reserved for pedestrian use and in which

All motorized traffic is prohibited. Some streets are partially pedestrianized, for instance during certain times such as evenings or weekends, while other, pedestrian-priority streets use Traffic Calming design strategies to control traffic speeds and volumes (Boyd 1998). According to (Elhamy, 2012) the pedestrian life Streets Are full of Life that enhance pedestrian design. These components convert the pedestrian life network in urban and suburban areas in to active well-designed commercial streets. Paving, street furniture landscaping and lighting make the public sidewalk a place where people want to participate and enhance 
the social interaction. These features create a sense of place identity on the street and are also important visual traffic calming measures. Furthermore, retail and restaurants increase the approach of pedestrian-friendly streets.

People also want to have their shopping in an environment where they can feel safe, not only safe from motor vehicle traffic, but safe from crime or other concerns that can affect personal security. Areas need to be well lit to encourage walking during evening hours. If the pedestrian system is not accessible, it is often not safe. For example, lack of access may cause wheelchair users to use the street rather than a poorly maintained sidewalk. Some populations may be at a higher risk of pedestrian crashes. Children under age 15 are the most overrepresented group in pedestrian crashes and people over age 65 have the most pedestrian Facilities,

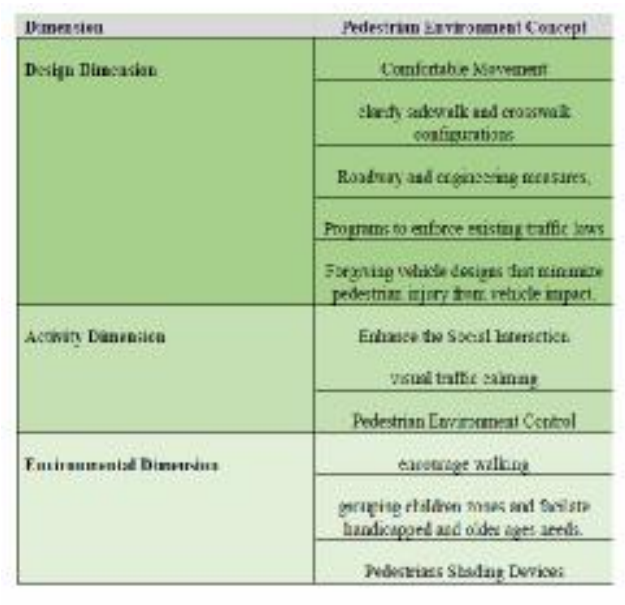

Therefore, it is especially important to provide facilities for grouping children zones and facilitate handicapped and older ages neers

Figure 8: Different theories that study Mixed of uses in the literature review and its relation with the sustainable commercial streets . Source: Author

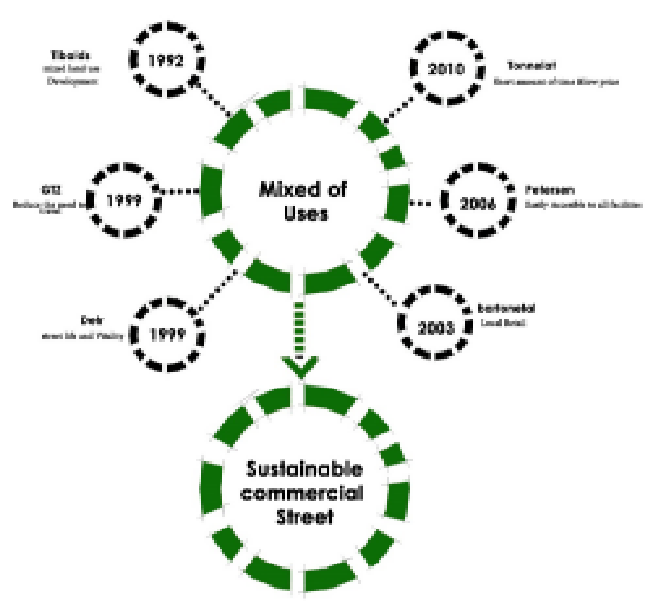

\subsection{Mixed of Uses as a concept}

Many urban designers now promote the advantages of traditional mixed-used streets. This has forced a consideration of how such streets might be designed and how they shape the urban fabric. According to Marshall $(2005 ; 2009)$ the urban structure, understood in a purely physical sense, is highly influenced by the structure of movement and access. The connective tissue (Marshall, 2005) of urban space is identified here with movement to and through spaces. An urban flux that carries social and cultural meaning where street patterns are the production of the geometry of movement and connectivity of places. 
While the sustainable UK Labour government strategy operates at a broad scale. At the local level, definitions of sustainability are harder to derive within the relatively limited context of a mixed-use street. Barton et al (2003), in their manual for new sustainable neighborhoods, propose a reinvention of the mixed-use high street. Their argument for its sustainability as a focus for local retail and commercial uses is based on the catchment area for a residential neighborhood enabling local residents to walk or cycle to the facilities. This provision of local services that are accessible by pedestrians Sustainability objectives.

Figure 9 : Different theories that study Accessibility in the literature review and its relation with the sustainable commercial streets .
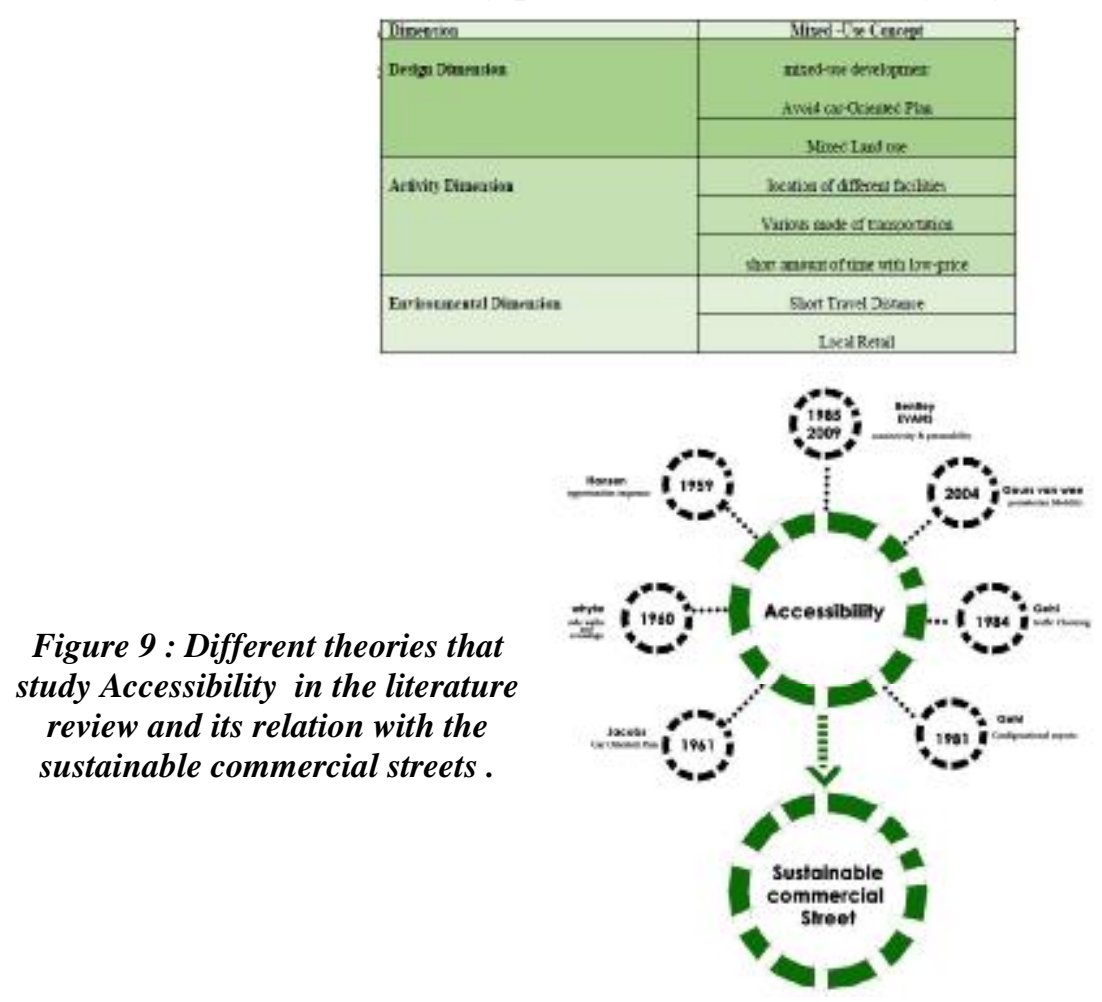

\subsection{Accessibility as a Concept}

Accessibility as a concept was studied in terms of vehicle movement. So, it was transportation activity that was mainly considered (Evans2009), which overlooked accessibility in favor of car mobility and ignored its true meaning, as such design focused on cars and their movement which led to unpleasant chaotic streets. Consequently, a new paradigm shift was required to link accessibility to its original meaning and the people centered approach rather than the carcentered approach appeared (floater,2014).

(Jacobs, 1961) Described all the surrounding streets design was based on a concept of "Car Oriented Plan" which is based on the car proportions, size and active space which affect all the street width and sidewalks in the planning Design process to fit all the car needs and this affect the cross distance and change the suitable distance for the pedestrians' users that directly affect the distance, time and Efforts that led to the decreasing of "pedestrian Mobility "in our streets. This mean that Accessibility term should be designed to serve all various users with different needs and to study the pedestrian, handicapped, children. Elder age users' needs to reach their target in the shortest distance and limited period of time without the less obstacles.

The Pedestrian Mobility as (Geurs, 2004) Studied that it is one of the accessible physical Design Dimension. Having Defined Nodes across the street is a main need for accessibility. They Guarantee the Recharging of Pedestrians ability, to continue their journey through the 
street. (Geurs, 2004) also, that Effort and time are important needed, the more the commercial street is accessible. (patxi, 2015) works on raising the individual's confidence in using the commercial street through Defining lanes and presence of Directional Signs.

The space accessibility would be more dynamic and safe along the same street directly movement in a commercial street for an example the distribution of the retail stores and the user would reach them in an easy way without any segregation External Elements. This would Force the users to place various and different activities either a commercial activity or even create a new approach for more and more new activities in the street within different scale of street either intimate or large scale for the Urban space. Moreover the site location of the urban elements and buildings should be selected and designed in such a manner that creates no obstacles and distortion for the view of residents of the surrounding buildings.

Figure 10 : Different theories that

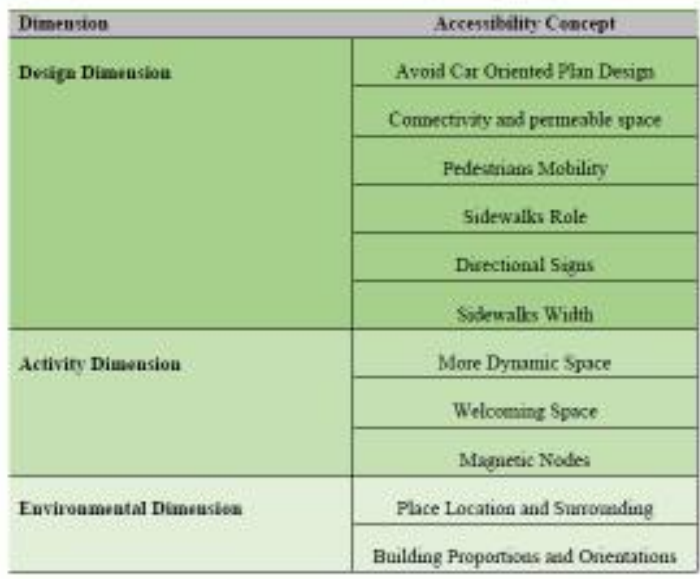
study Safety in the literature review and its relation with the sustainable commercial streets .

\subsection{Safety as a Concept}

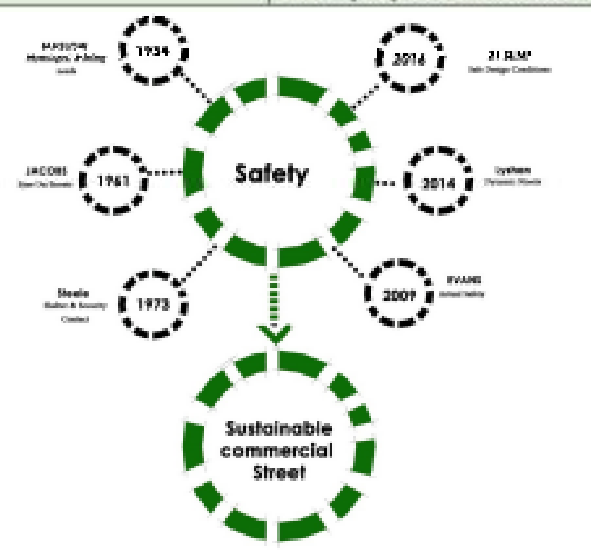

Safety is a substantial need in commercial streets where its absence prevents the occurrence of a dynamic life of the street and keeps the street far from achieving high standards. (Lyshan,2014),An empty street for the users is like an empty painting frame, dangerous Space for users . Such surveillance requires a big number of stores and public places scattered along the sidewalk; especially night used places such as shops and stores that contribute to the safety and security, these sidewalks gain actual reasons for its usage by both the residents and the random users of these enterprises.

The role of natural day light is highly important in the street safety, Bright street lights gain extra value for the comfort and safety they offer to people, therefore increasing the contribution of these people as a surveillance eye on the street. However, streetlights cannot perform on its own. Shocking crimes still occur in well-lighted stations and places. And 
TOWARDS SUSTAINABLE COMMERCIAL STREETS

unless there are eyes that overlooks these places holding the unconscious assurance of street support and safety these crimes will still occur (J. Jacobs, 1961). 

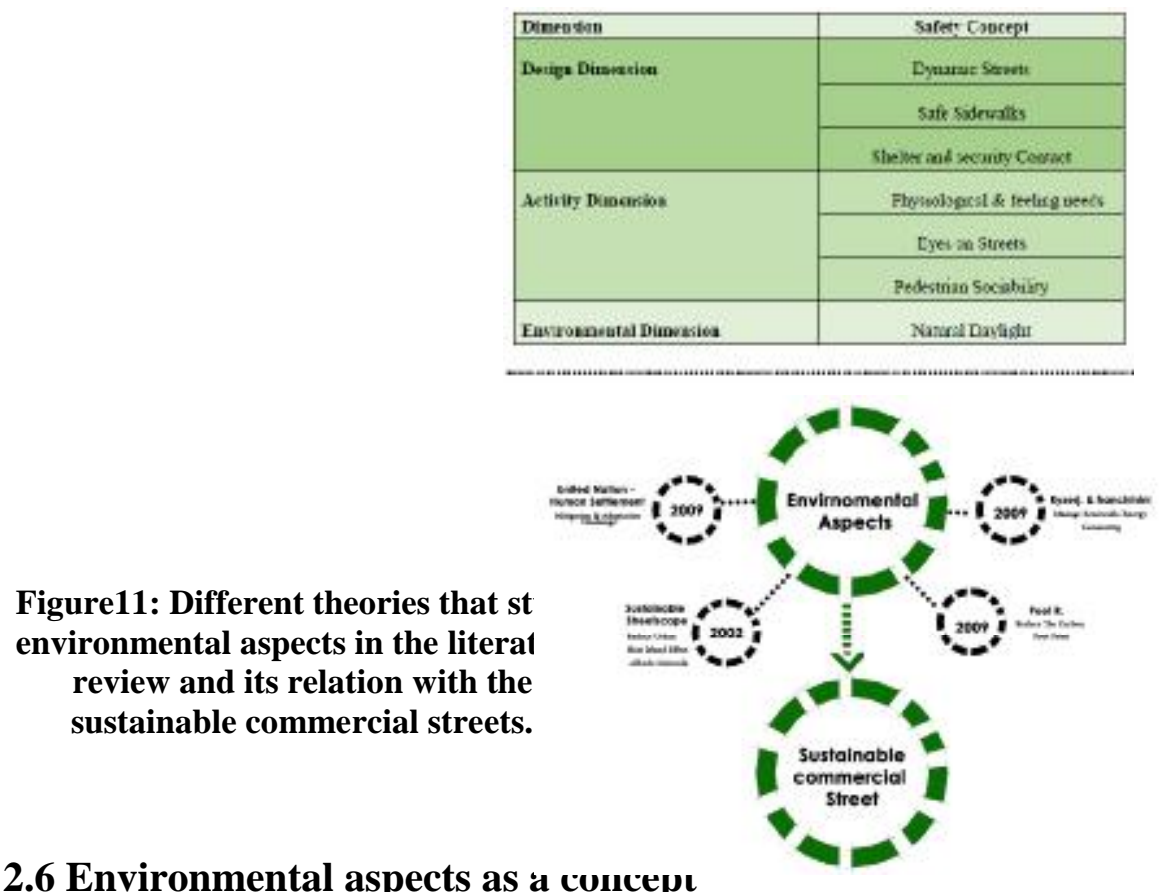

\subsection{Environmental aspects as à concept}

In our Community and Urban Spaces Environment and energy are two sides of the same coin. The Streets are the major consumers of fossil fuels which have risen from $4 \%$ of global energy consumption in the early 1900 s to over $86 \%$ at present time. For all these reasons, Streets play an excessive role in greenhouse gas emissions. Mitigating $\mathrm{CO} 2$ emissions and adapting to climate change are essential at the scale of a street level. in Egypt consumes $19.2 \%$ of the total energy consumption and about $39.1 \%$ of the total electricity consumption, which produces $10 \%$ of the total CO2 emission, (Ministry of Energy and Electricity, 2003). On the other hand, Egypt has a good potential of renewable resources including solar and wind power. Thus, renewable energy technologies can be considered as an integral part of the Egyptian energy policy framework. (Gihan Mosaad Hannallah, 2014).

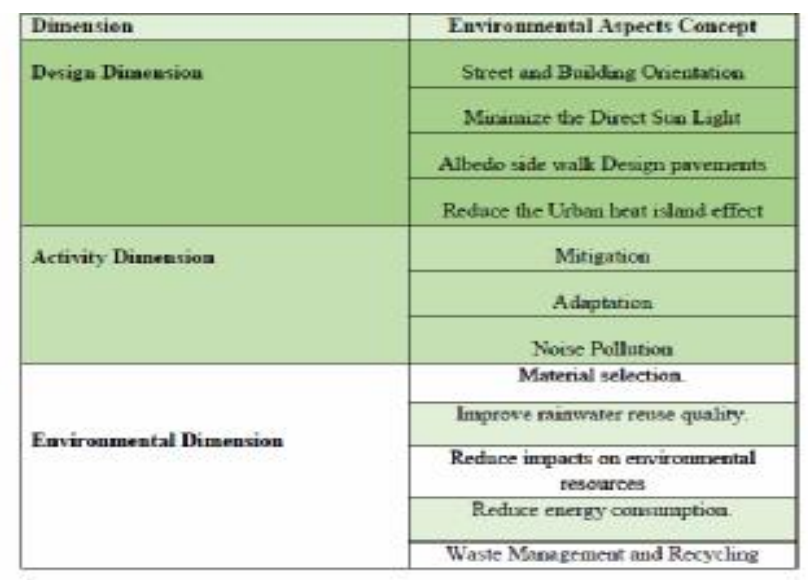

\subsection{Extracting Concepts:}

This Extracting Concepts and Dimensions formulates an accumulative meaning of sustainability in the commercial streets. Which is the afordance of the physical active design within the surrounding environmental to attract and support Pedestrians activities accompanied by providing investments and economic Growth. 
Theorists who studied Sustainability through time linked the state of sustainability to complicated Concepts. The research arrived at the six concluded concepts that repeated by theorists through different eras in their study of the sustainability in the streets, it become clearer through the analytical examples that all the six concepts must be achieved at different various levels, towards the state of sustainability target

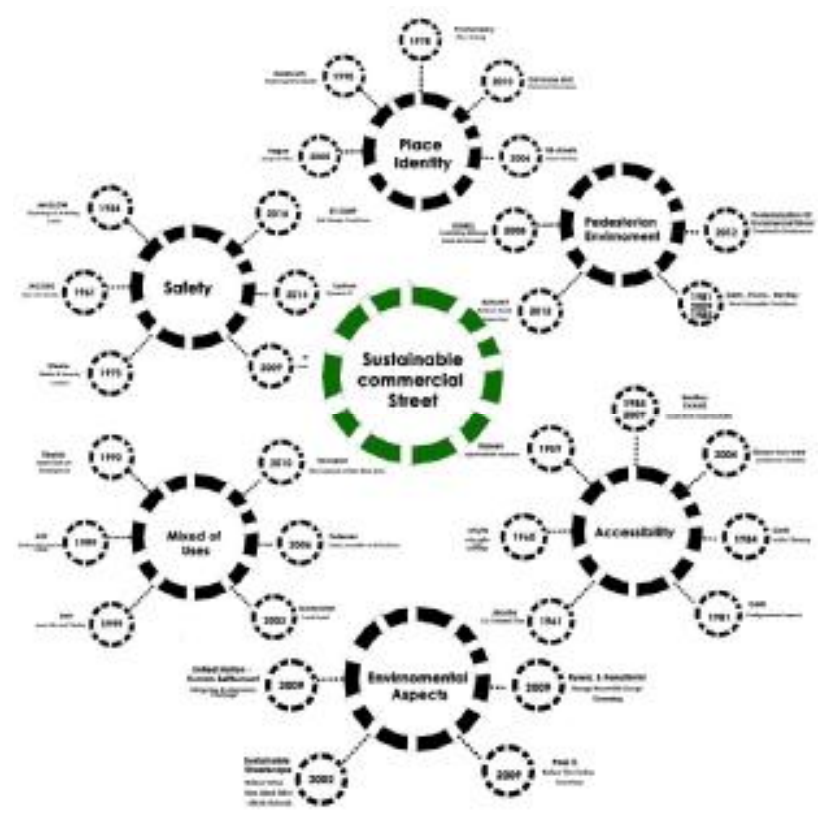

Figure 12 : link between the six concepts of sustainable commercial streets

Later, the research considered these six concepts as the Concepts of Sustainable Commercial Streets. As they all imperative to reach the state of sustainability, they are: place identity, Mixed of Uses, Pedestrian Environment, Accessibility, Safety and The Environmental Aspects. Coordinating each concept's indicator in design, activity and Environmental Dimensions shaped the Reference table. This table is set into two axial. It consists horizontally from the six concepts and vertically from the three dimension. 


\begin{tabular}{|c|c|c|c|c|c|c|}
\hline \multicolumn{7}{|c|}{ Concepts of Sustainable Commercial Streets } \\
\hline Dim. & Place Identity & $\begin{array}{c}\text { Mixed } \\
\text { Uses } \\
\end{array}$ & $\begin{array}{c}\text { Pedestrian } \\
\text { Environment }\end{array}$ & Accessibility & Safety & $\begin{array}{c}\text { Environmental } \\
\text { aspects }\end{array}$ \\
\hline \multirow{6}{*}{ 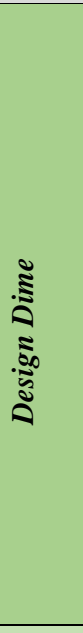 } & $\begin{array}{l}\text { Image and } \\
\text { Identity }\end{array}$ & $\begin{array}{c}\text { Mixed-use } \\
\text { development }\end{array}$ & $\begin{array}{c}\text { Comfortable } \\
\text { Movement }\end{array}$ & $\begin{array}{c}\text { Avoid Car } \\
\text { Oriented Plan } \\
\text { Design }\end{array}$ & $\begin{array}{c}\text { Dynamic } \\
\text { Streets }\end{array}$ & $\begin{array}{l}\text { Street and Building } \\
\text { Orientation }\end{array}$ \\
\hline & $\begin{array}{l}\text { Symbolic } \\
\text { Meaning }\end{array}$ & $\begin{array}{l}\text { Avoid car- } \\
\text { Oriented } \\
\text { Plan }\end{array}$ & $\begin{array}{c}\text { Clarify sidewalk and } \\
\text { crosswalk } \\
\text { configurations }\end{array}$ & $\begin{array}{c}\text { Connectivity } \\
\text { and permeable } \\
\text { space }\end{array}$ & $\begin{array}{c}\text { Safe } \\
\text { Sidewalks }\end{array}$ & $\begin{array}{c}\text { Minimize the Direct } \\
\text { Sun Light }\end{array}$ \\
\hline & $\begin{array}{c}\text { Historic } \\
\text { districts } \\
\text { streets }\end{array}$ & $\begin{array}{l}\text { Mixed Land } \\
\text { use }\end{array}$ & $\begin{array}{l}\text { Roadway and } \\
\text { engineering } \\
\text { measures, }\end{array}$ & $\begin{array}{l}\text { Pedestrians } \\
\text { Mobility }\end{array}$ & $\begin{array}{l}\text { Shelter and } \\
\text { security } \\
\text { Contact }\end{array}$ & $\begin{array}{l}\text { Albedo side walk } \\
\text { Design pavements }\end{array}$ \\
\hline & $\begin{array}{l}\text { heritage } \\
\text { significance }\end{array}$ & & $\begin{array}{l}\text { Programs to enforce } \\
\text { existing traffic laws }\end{array}$ & Sidewalks Role & & $\begin{array}{l}\text { Reduce the Urban } \\
\text { heat island effect }\end{array}$ \\
\hline & & & $\begin{array}{c}\text { Forgiving vehicle } \\
\text { designs that } \\
\text { minimize pedestrian } \\
\text { injury } \\
\end{array}$ & $\begin{array}{l}\text { Directional } \\
\text { Signs }\end{array}$ & & \\
\hline & & & & $\begin{array}{l}\text { Sidewalks } \\
\text { Width }\end{array}$ & & \\
\hline \multirow{3}{*}{ 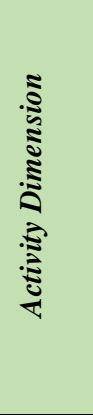 } & $\begin{array}{c}\text { Historical } \\
\text { dimensions }\end{array}$ & $\begin{array}{l}\text { location of } \\
\text { different } \\
\text { facilities }\end{array}$ & $\begin{array}{l}\text { Enhance the Social } \\
\text { Interaction }\end{array}$ & $\begin{array}{l}\text { More Dynamic } \\
\text { Space }\end{array}$ & $\begin{array}{l}\text { Physiologic } \\
\text { al \& } \\
\text { feeling } \\
\text { needs } \\
\end{array}$ & Mitigation \\
\hline & $\begin{array}{c}\text { Monumental } \\
\text { memory }\end{array}$ & $\begin{array}{c}\text { Various mode } \\
\text { of } \\
\text { transportatio } \\
n \\
\end{array}$ & $\begin{array}{l}\text { visual traffic } \\
\text { calming }\end{array}$ & $\begin{array}{l}\text { Welcoming } \\
\text { Space }\end{array}$ & $\begin{array}{c}\text { Eyes on } \\
\text { Streets }\end{array}$ & Adaptation \\
\hline & & $\begin{array}{c}\text { short amount } \\
\text { of time with } \\
\text { low-price }\end{array}$ & $\begin{array}{c}\text { Pedestrian } \\
\text { Environment } \\
\text { Control }\end{array}$ & Magnetic Nodes & $\begin{array}{l}\text { Pedestrian } \\
\text { Sociability }\end{array}$ & Nosie Pollution \\
\hline \multirow{5}{*}{ 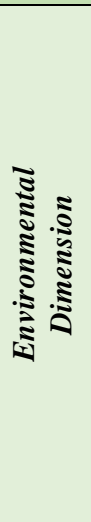 } & Preserving & $\begin{array}{l}\text { Short Travel } \\
\text { Distance }\end{array}$ & Encourage walking & $\begin{array}{c}\text { Place Location } \\
\text { and } \\
\text { Surrounding }\end{array}$ & $\begin{array}{l}\text { Natural } \\
\text { Daylight }\end{array}$ & Material selection. \\
\hline & & Local Retail & $\begin{array}{l}\text { Grouping children } \\
\text { zones and facilitate } \\
\text { handicapped and } \\
\text { older ages needs }\end{array}$ & $\begin{array}{c}\text { Building } \\
\text { Proportions and } \\
\text { Orientations }\end{array}$ & & $\begin{array}{l}\text { Improve rainwater } \\
\text { reuse quality. }\end{array}$ \\
\hline & & & $\begin{array}{c}\text { Pedestrians Shading } \\
\text { Devices }\end{array}$ & & & $\begin{array}{c}\text { Reduce impacts on } \\
\text { environmental } \\
\text { resources }\end{array}$ \\
\hline & & & & & & $\begin{array}{l}\text { Reduce energy } \\
\text { consumption. }\end{array}$ \\
\hline & & & & & & $\begin{array}{c}\text { Waste Management } \\
\text { and Recycling }\end{array}$ \\
\hline
\end{tabular}

\section{SUSTAINABLE COMMERCIAL STREET IN PRACTICE (TESTING METHODOLOGY)}

This Analysis on Two Commercial Streets, an international example and a local one, their Aim are to examine the appliance of the Six Developed in the previous section. the need of the state of sustainability in the Commercial Streets through the six concepts together to achieve the state of sustainability. Two steps were taken in consideration in analyzing the two examples. First, illustrates the Reasons of being in the state of Sustainability. Secondly, Determines the Concepts Each Street Followed to provide the State of sustainability through the indicators Design, Activity and Environmental Dimensions for the Commercial Streets.

\subsection{First Examine Case: King-Victoria Transit Hub Street.}

The King-Victoria Transit Hub, is known as the Central intermodal transportation Station, terminal in Kitchener, Ontario, Canada. It is a planned as the It will serve the area of Ion light rail line, GO Transit trains and Via Rail. The station will replace the current Kitchener railway station and the 
intercity bus services which currently depart from Charles Street will be relocated to the new proposed new terminal.

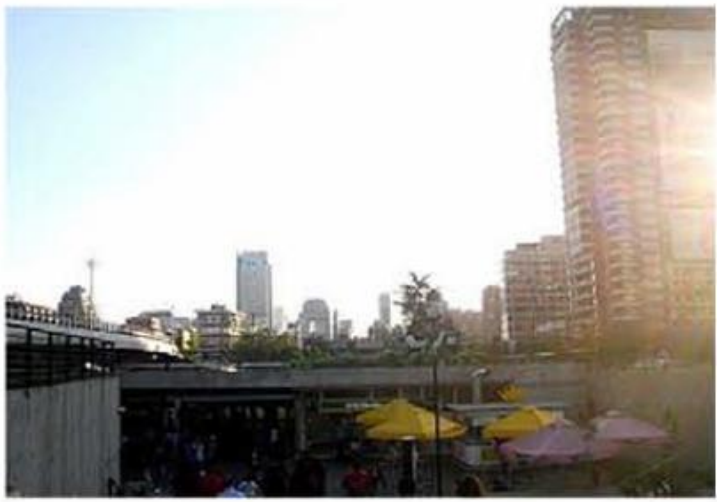

Figure 1 the day light gives the underground space safe feeling and natural day light

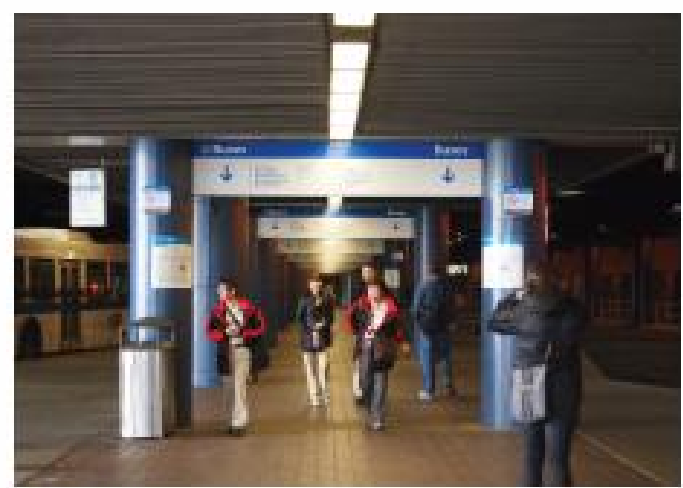

An off-street bus terminal that serves intercity buses, GO Transit buses, and GRT buses that terminate at the Transit Hub. The terminal could also allow for MobilityPLUS pick-ups and drop-offs

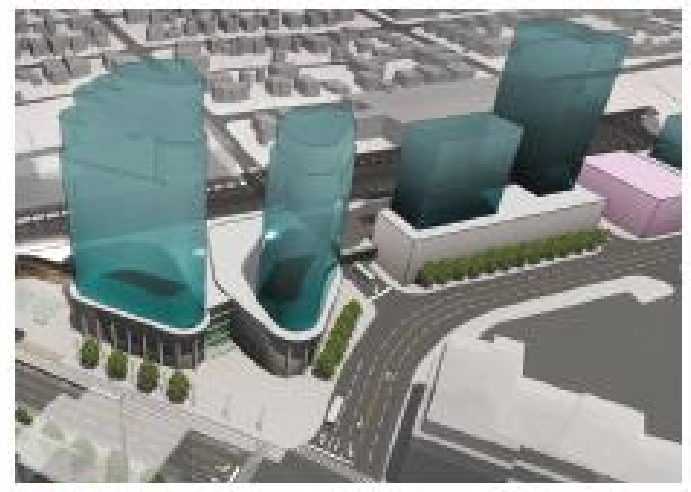

Mixed-use development integrated with the Transit Hub, with office, retail, residential, and other uses at a higher intensity forming a landmark in downtown Kitchener

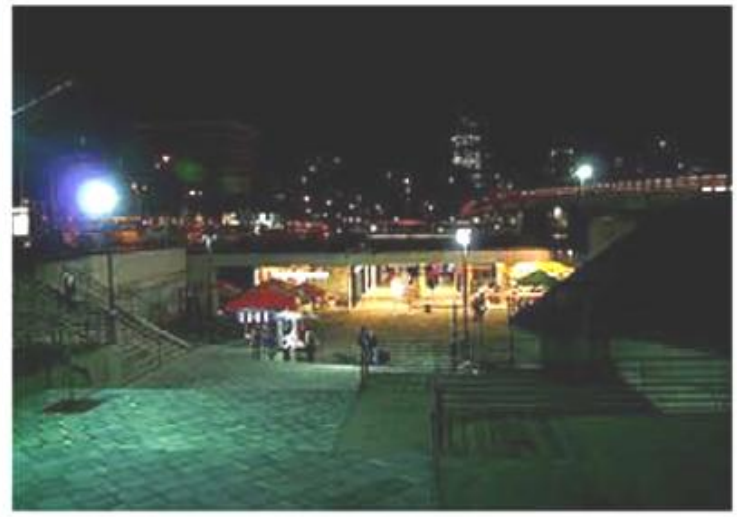

Figure 2 also the night commercial light gives the same safe feeling space

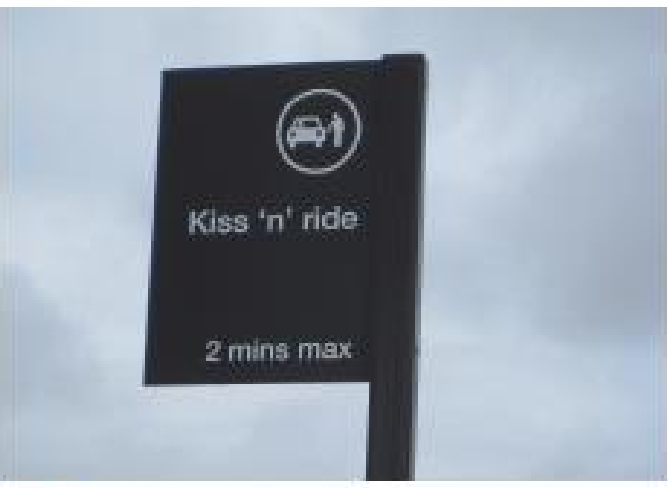

Passenger pick-up and drop-off (oKiss and Ride) could be facilitated on the upper (track) level from Duke street, providing direct access to the rail platforms

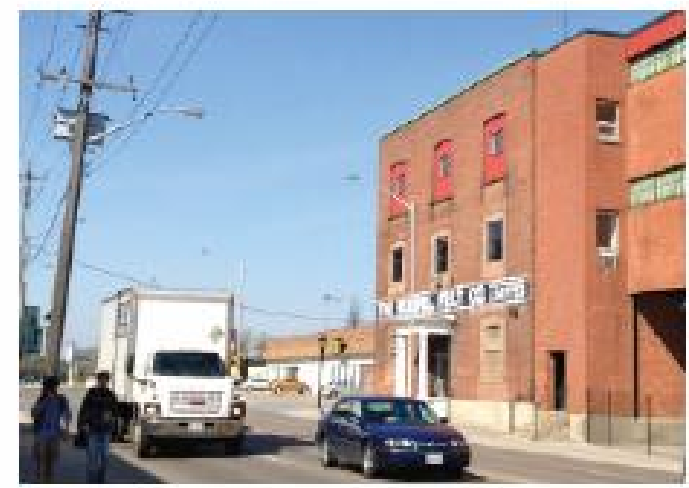

The original Rumpel Felt Building has cultural

8 heritage value and is intended to be adaptively reused as part of the Transit Hub plan. 


\subsubsection{Examining the extracting Concepts with the Three Dimensions}

Table1 the Appraisal of the Referential Set in King Victoria Hub Street Source: Researcher

\begin{tabular}{|c|c|c|c|c|c|c|c|c|c|c|c|c|}
\hline \multicolumn{13}{|c|}{ Concepts of Sustainable Commercial Streets } \\
\hline Dim. & Place Identity & & $\begin{array}{c}\text { Mixed } \\
\text { Uses }\end{array}$ & & $\begin{array}{c}\text { Pedestrian } \\
\text { Environment }\end{array}$ & & Accessibility & & Safety & & $\begin{array}{c}\text { Environmenta } \\
\text { aspects }\end{array}$ & \\
\hline \multirow{6}{*}{ 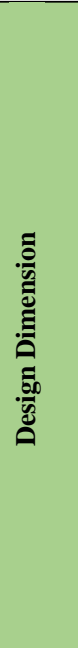 } & $\begin{array}{l}\text { Image and } \\
\text { Identity }\end{array}$ & G & $\begin{array}{l}\text { Mixed-use } \\
\text { development }\end{array}$ & G & $\begin{array}{l}\text { Comfortable } \\
\text { Movement }\end{array}$ & G & $\begin{array}{c}\text { Avoid Car } \\
\text { Oriented Plan } \\
\text { Design }\end{array}$ & $\mathrm{F}$ & $\begin{array}{c}\text { Dynamic } \\
\text { Streets }\end{array}$ & G & $\begin{array}{l}\text { Street and } \\
\text { Building } \\
\text { Orientation }\end{array}$ & $\mathrm{F}$ \\
\hline & $\begin{array}{l}\text { Symbolic } \\
\text { Meaning }\end{array}$ & G & $\begin{array}{l}\text { Avoid car- } \\
\text { Oriented Plan }\end{array}$ & $\mathrm{F}$ & $\begin{array}{l}\text { Clarify sidewalk } \\
\text { and crosswalk } \\
\text { configurations }\end{array}$ & $\mathrm{F}$ & $\begin{array}{l}\text { Connectivity and } \\
\text { permeable } \\
\text { space }\end{array}$ & G & $\begin{array}{c}\text { Safe } \\
\text { Sidewalks }\end{array}$ & G & $\begin{array}{l}\text { Minimize the } \\
\text { Direct Sun } \\
\text { Light }\end{array}$ & $\mathrm{G}$ \\
\hline & $\begin{array}{c}\text { Historic } \\
\text { districts streets }\end{array}$ & $\mathrm{F}$ & Mixed Land use & G & $\begin{array}{c}\text { Roadway and } \\
\text { engineering } \\
\text { measures, }\end{array}$ & $\mathrm{F}$ & $\begin{array}{c}\text { Pedestrians } \\
\text { Mobility }\end{array}$ & G & $\begin{array}{c}\text { Shelter and } \\
\text { security } \\
\text { Contact }\end{array}$ & G & $\begin{array}{c}\text { Albedo side } \\
\text { walk Design } \\
\text { pavements }\end{array}$ & $\mathrm{G}$ \\
\hline & $\begin{array}{l}\text { heritage } \\
\text { significance }\end{array}$ & $\mathrm{F}$ & & & $\begin{array}{l}\text { Programs to } \\
\text { enforce existing } \\
\text { traffic laws }\end{array}$ & $\mathrm{P}$ & Sidewalks Role & $\mathrm{G}$ & & & $\begin{array}{c}\text { Reduce the } \\
\text { Urban heat } \\
\text { island effect }\end{array}$ & $\mathrm{F}$ \\
\hline & & & & & $\begin{array}{c}\text { Forgiving vehicle } \\
\text { designs that } \\
\text { minimize } \\
\text { pedestrian injury }\end{array}$ & $\mathrm{F}$ & Directional Signs & G & & & & \\
\hline & & & & & & & Sidewalks Width & G & & & & \\
\hline \multirow{3}{*}{ 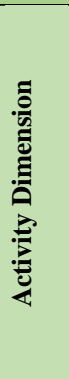 } & $\begin{array}{c}\text { Historical } \\
\text { dimensions }\end{array}$ & $\mathrm{F}$ & $\begin{array}{l}\text { location of } \\
\text { different } \\
\text { facilities }\end{array}$ & G & $\begin{array}{c}\text { Enhance the } \\
\text { Social Interaction }\end{array}$ & G & $\begin{array}{c}\text { More Dynamic } \\
\text { Space }\end{array}$ & G & $\begin{array}{c}\text { Physiological } \\
\& \text { feeling } \\
\text { needs }\end{array}$ & G & Mitigation & $\mathrm{F}$ \\
\hline & $\begin{array}{c}\text { Monumental } \\
\text { memory }\end{array}$ & $\mathrm{P}$ & $\begin{array}{l}\text { Various mode of } \\
\text { transportation }\end{array}$ & G & $\begin{array}{c}\text { visual traffic } \\
\text { calming }\end{array}$ & G & $\begin{array}{l}\text { Welcoming } \\
\text { Space }\end{array}$ & G & $\begin{array}{l}\text { Eyes on } \\
\text { Streets }\end{array}$ & G & Adaptation & $\mathrm{F}$ \\
\hline & & & $\begin{array}{l}\text { short amount of } \\
\text { time with low- } \\
\text { price }\end{array}$ & G & $\begin{array}{l}\text { Pedestrian } \\
\text { Environment } \\
\text { Control }\end{array}$ & G & Magnetic Nodes & G & $\begin{array}{l}\text { Pedestrian } \\
\text { Sociability }\end{array}$ & G & & \\
\hline \multirow{5}{*}{ 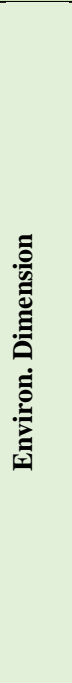 } & Preserving & $\mathrm{F}$ & $\begin{array}{c}\text { Short Travel } \\
\text { Distance }\end{array}$ & G & encourage walking & G & $\begin{array}{l}\text { Place Location \& } \\
\text { Surrounding }\end{array}$ & G & $\begin{array}{c}\text { Natural } \\
\text { Daylight }\end{array}$ & G & $\begin{array}{l}\text { Material } \\
\text { selection. }\end{array}$ & $\mathrm{G}$ \\
\hline & & & Local Retail & $\mathrm{F}$ & $\begin{array}{c}\text { Facilitate children } \\
\text { and handicapped } \\
\text { zones \& }\end{array}$ & $\mathrm{F}$ & $\begin{array}{c}\text { Building } \\
\text { Proportions and } \\
\text { Orientations }\end{array}$ & $\mathrm{F}$ & & & $\begin{array}{c}\text { Improve } \\
\text { rainwater } \\
\text { reuse quality. }\end{array}$ & $\mathrm{F}$ \\
\hline & & & & & $\begin{array}{c}\text { Pedestrians } \\
\text { Shading Devices }\end{array}$ & G & & & & & $\begin{array}{c}\text { Reduce } \\
\text { Environment } \\
\text { al Impacts }\end{array}$ & $\mathrm{G}$ \\
\hline & & & & & & & & & & & $\begin{array}{c}\text { Reduce } \\
\text { energy } \\
\text { consumption }\end{array}$ & $\mathrm{F}$ \\
\hline & & & & & & & & & & & $\begin{array}{c}\text { Waste } \\
\text { Management } \\
\text { and } \\
\text { Recycling }\end{array}$ & $\mathrm{G}$ \\
\hline
\end{tabular}

Key Table $\quad$ Good $=\mathrm{G} \quad$ Fair $=F \quad$ Poor $=\mathrm{P}$ 


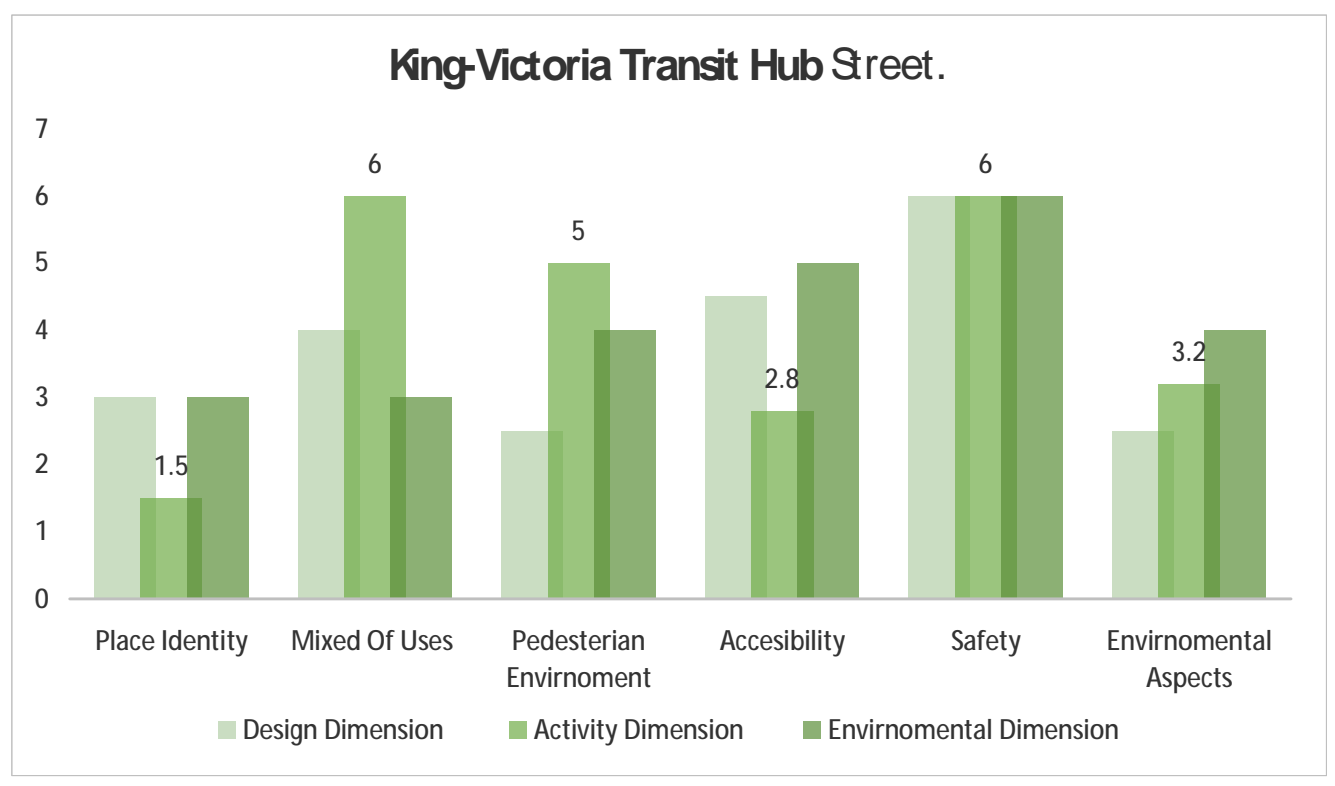

Figure Chart Shows the Percentage Of sustainable Target in the Street source: The Researcher

\subsection{Second Examine Case: Al Gouna Down Town Commercial Street.}

ALGouna Downtown Commercial Public Space area; where most of the commercial facilities are found. Which is mainly a pedestrian square full of outdoor cafes and Open restaurants, developed and owned by Orascom Hotels and Development (OHD), da ng from about 1990 in the Gouna Village.

The Space planning and design is compact and is characterized by the visibility of its entrance. It has been taken into consideration that the character of the city development is derived from the traditional rural Egyptian context Buildings are covered by domes and vaults and streets with Porous paving materials or a bright asphalt in order to provide the interior with moderate climate, (Nady,R. 2008).

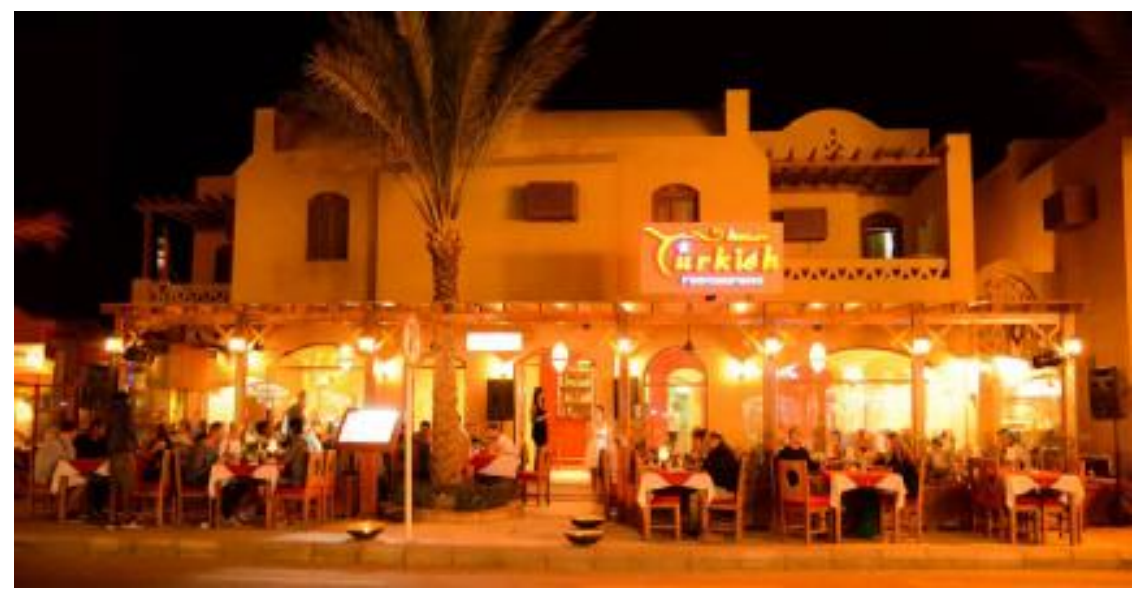

Figure : Down town cafeterias Source: Algouna state of mind magazine 

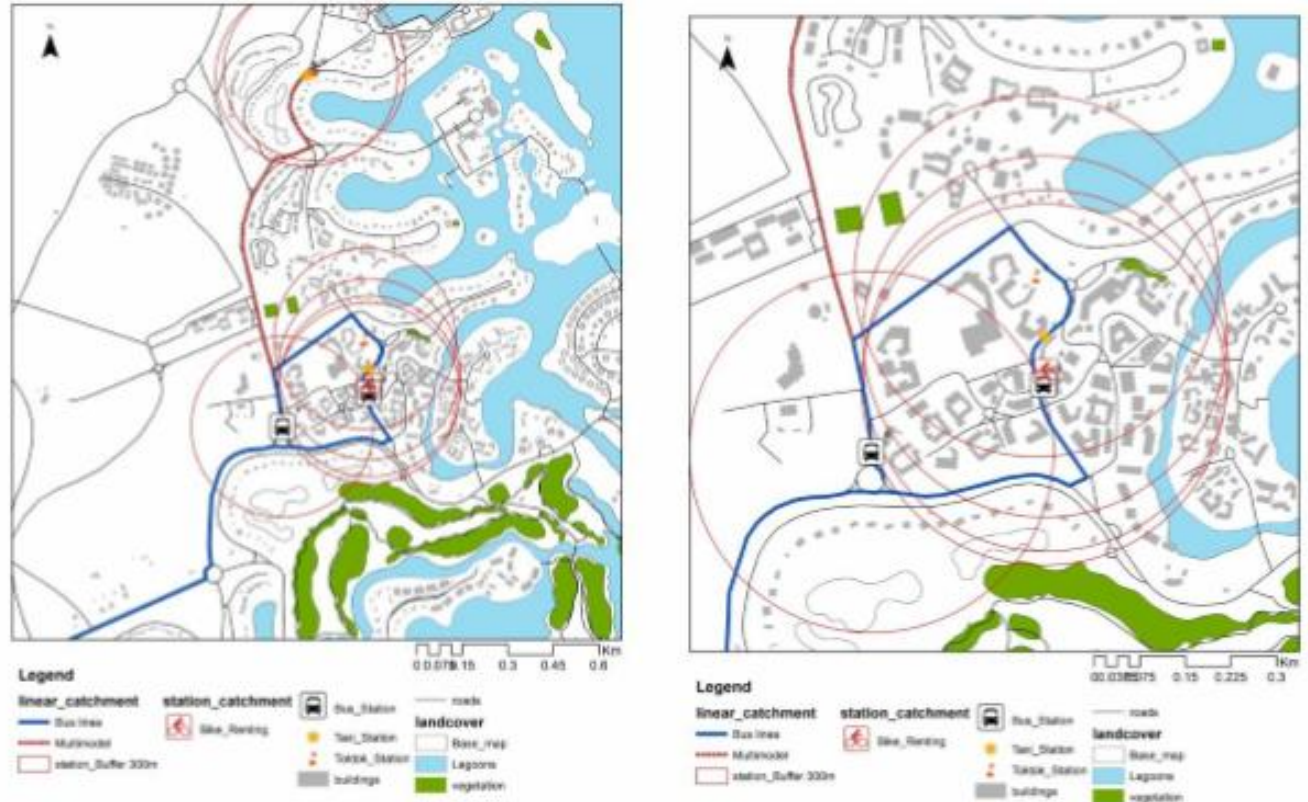

Figure 3 Accessibility map for AlGouna source: Ahmed Khalil

\subsubsection{Examining the extracting Concepts with the Three Dimensions}

Table: Shows the Appraisal of the Referential Set in King Victoria Hub Street Source: Researcher

\begin{tabular}{|c|c|c|c|c|c|c|c|c|c|c|c|c|}
\hline \multicolumn{13}{|c|}{ Concepts of Sustainable Commercial Streets } \\
\hline Dim & $\begin{array}{c}\text { Place } \\
\text { Identity }\end{array}$ & & $\begin{array}{l}\text { Mixed } \\
\text { Uses }\end{array}$ & & $\begin{array}{l}\text { Pedestrian } \\
\text { Environment }\end{array}$ & & ccessibility & & Safety & & $\begin{array}{l}\text { nvironment } \\
\text { aspects }\end{array}$ & \\
\hline \multirow{6}{*}{ 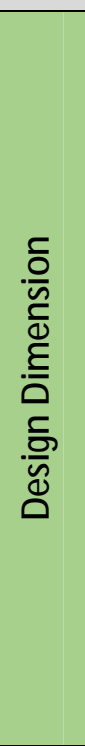 } & $\begin{array}{l}\text { Image and } \\
\text { Identity }\end{array}$ & G & $\begin{array}{c}\text { Mixed-use } \\
\text { development }\end{array}$ & G & $\begin{array}{c}\text { Comfortable } \\
\text { Movement }\end{array}$ & G & $\begin{array}{l}\text { Avoid Car } \\
\text { Oriented Plan } \\
\text { Design }\end{array}$ & $\mathrm{F}$ & $\begin{array}{l}\text { Dynamic } \\
\text { Streets }\end{array}$ & G & $\begin{array}{c}\text { Street and } \\
\text { Building } \\
\text { Orientation }\end{array}$ & $P$ \\
\hline & $\begin{array}{l}\text { Symbolic } \\
\text { Meaning }\end{array}$ & G & $\begin{array}{l}\text { Avoid car- } \\
\text { Oriented Plan }\end{array}$ & $\mathrm{F}$ & $\begin{array}{l}\text { Clarify sidewalk } \\
\text { and crosswalk } \\
\text { configurations }\end{array}$ & G & $\begin{array}{l}\text { Connectivity } \\
\text { and permeable } \\
\text { space }\end{array}$ & G & $\begin{array}{c}\text { Safe } \\
\text { Sidewalks }\end{array}$ & G & $\begin{array}{l}\text { Minimize } \\
\text { the Direct } \\
\text { Sun Light }\end{array}$ & $\mathrm{F}$ \\
\hline & $\begin{array}{l}\text { Historic } \\
\text { districts } \\
\text { streets }\end{array}$ & $\mathrm{p}$ & M ixed Land use & G & $\begin{array}{l}\text { Roadway and } \\
\text { engineering } \\
\text { measures, }\end{array}$ & $\mathrm{F}$ & $\begin{array}{c}\text { Pedestrians } \\
\text { Mobility }\end{array}$ & G & $\begin{array}{l}\text { Shelter and } \\
\text { security } \\
\text { Contact }\end{array}$ & G & $\begin{array}{c}\text { Albedo side } \\
\text { walk Design } \\
\text { pavements }\end{array}$ & $P$ \\
\hline & $\begin{array}{l}\text { heritage } \\
\text { significance }\end{array}$ & $p$ & & & $\begin{array}{l}\text { Programs to } \\
\text { enforce existing } \\
\text { traffic laws }\end{array}$ & $\mathrm{F}$ & Sidewalks Role & G & & & $\begin{array}{c}\text { Reduce the } \\
\text { Urban heat } \\
\text { island effect }\end{array}$ & $P$ \\
\hline & & & & & $\begin{array}{l}\text { Forgiving vehicle } \\
\text { designs that } \\
\text { minimize } \\
\text { pedestrian injury }\end{array}$ & $\mathrm{F}$ & $\begin{array}{l}\text { Directional } \\
\text { Signs }\end{array}$ & G & & & & \\
\hline & & & & & & & $\begin{array}{l}\text { Sidewalks } \\
\text { Width }\end{array}$ & $\mathrm{F}$ & & & & \\
\hline \multirow{3}{*}{ 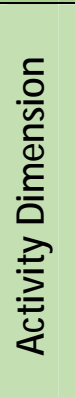 } & $\begin{array}{c}\text { Historical } \\
\text { dimensions }\end{array}$ & $p$ & $\begin{array}{l}\text { location of } \\
\text { different } \\
\text { facilities }\end{array}$ & G & $\begin{array}{l}\text { Enhance the } \\
\text { Social } \\
\text { Interaction }\end{array}$ & $\mathrm{F}$ & $\begin{array}{l}\text { More Dynamic } \\
\text { Space }\end{array}$ & G & $\begin{array}{l}\text { Physiologica } \\
\text { I \& feeling } \\
\text { needs }\end{array}$ & G & Mitigation & $\mathrm{F}$ \\
\hline & $\begin{array}{l}\text { Monument } \\
\text { al memory }\end{array}$ & $P$ & $\begin{array}{c}\text { Various mode } \\
\text { of } \\
\text { transportation }\end{array}$ & G & $\begin{array}{l}\text { visual traffic } \\
\text { calming }\end{array}$ & $\mathrm{F}$ & $\begin{array}{l}\text { Welcoming } \\
\text { Space }\end{array}$ & G & $\begin{array}{l}\text { Eyes on } \\
\text { Streets }\end{array}$ & G & Adaptation & $\mathrm{F}$ \\
\hline & & & $\begin{array}{l}\text { short amount } \\
\text { of time with } \\
\text { low-price }\end{array}$ & $\mathrm{F}$ & $\begin{array}{l}\text { Pedestrian } \\
\text { Environment } \\
\text { Control }\end{array}$ & G & $\begin{array}{l}\text { Magnetic } \\
\text { Nodes }\end{array}$ & G & $\begin{array}{l}\text { Pedestrian } \\
\text { Sociability }\end{array}$ & G & & \\
\hline
\end{tabular}




\begin{tabular}{|c|c|c|c|c|c|c|c|c|c|c|c|c|}
\hline \multirow{5}{*}{ 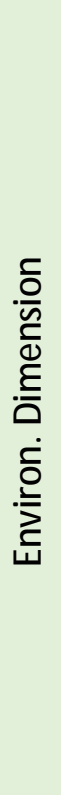 } & Preserving & $F$ & $\begin{array}{c}\text { Short Travel } \\
\text { Distance }\end{array}$ & G & $\begin{array}{l}\text { encourage } \\
\text { walking }\end{array}$ & G & $\begin{array}{l}\text { Place Location } \\
\& \text { Surrounding }\end{array}$ & G & $\begin{array}{l}\text { Natural } \\
\text { Daylight }\end{array}$ & G & $\begin{array}{l}\text { Material } \\
\text { selection. }\end{array}$ & G \\
\hline & & & Local Retail & G & $\begin{array}{c}\text { Facilitate } \\
\text { children and } \\
\text { handicapped } \\
\text { zones \& }\end{array}$ & $F$ & $\begin{array}{c}\text { Building } \\
\text { Proportions } \\
\text { and } \\
\text { Orientations }\end{array}$ & G & & & $\begin{array}{c}\text { Improve } \\
\text { rainwater } \\
\text { reuse } \\
\text { quality. }\end{array}$ & $F$ \\
\hline & & & & & $\begin{array}{c}\text { Pedestrians } \\
\text { Shading Devices }\end{array}$ & G & & & & & $\begin{array}{c}\text { Reduce } \\
\text { Environmen } \\
\text { tal Impacts }\end{array}$ & $F$ \\
\hline & & & & & & & & & & & $\begin{array}{c}\text { Reduce } \\
\text { energy } \\
\text { consumptio } \\
n\end{array}$ & G \\
\hline & & & & & & & & & & & $\begin{array}{c}\text { Waste } \\
\text { Managemen } \\
\mathrm{t} \text { and } \\
\text { Recycling }\end{array}$ & G \\
\hline
\end{tabular}

$\begin{array}{llll}\text { Key Table } & \text { Good }=\mathrm{G} & \text { Fair }=\mathrm{F} & \text { Poor }=\end{array}$

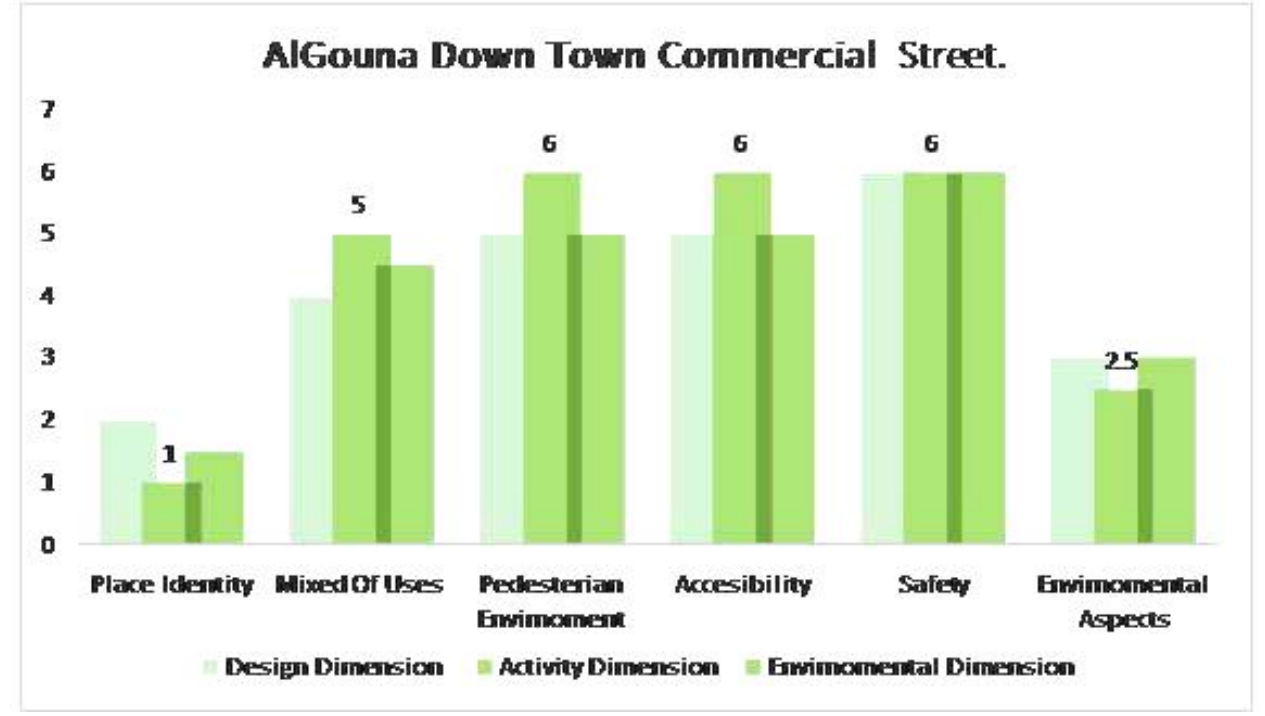

\section{Results Discussion:}

Figure: Chart Shows the Percentage Of sustainable Target in the Street source: The Researcher

\subsection{King Victoria Results and Discussion:}

\section{KINA G-YCTORLA ITRANMT HUR STREET.}

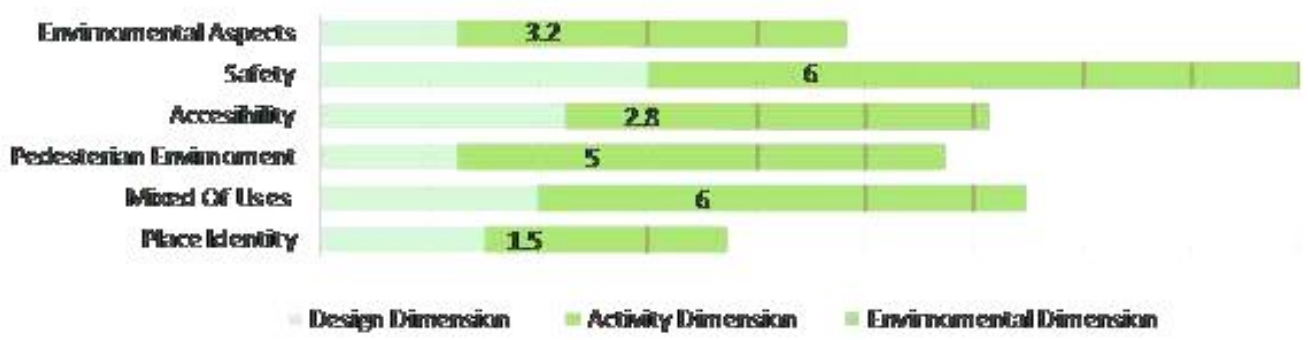

Figure: Chart Shows the Percentage Of sustainable Target in the Street source: Researcher 
From the evaluation Results in King Victoria transit hub Street, the sustainable achievement is quiet fine as the six concepts are presented by a constant rate , as the "Mixed uses " is presented by more than an activity in this active travelling area with various method of transportation in the street which encourage more people and more activity to be held in the street , while the "safety " method is also fine as the concept of" eyes on streets" were been applied through the presence of passerby and shop owners most of the time which give the street a self-safety use. In the other hand the place identity where been naturally present as the historical train station is located in the street. While the "Accessibility" and "pedestrian Environment "where been facilitated during the design development and provide a special underground bridge for the travelers and pedestrian from the train station to all the other street nodes with a clear and well-lighted sidewalk for all the users. What is worth to mention that the previous 5 dimensions are represented by a fine percent but the Environmental aspects are the lowest evaluations in the street as they concentrated only on the recycling methods but ignored any way for using a water management system that can be very useful especially in this zone of the street especially in winter.

\subsection{Algouna Down Town Street Results and Discussion:}

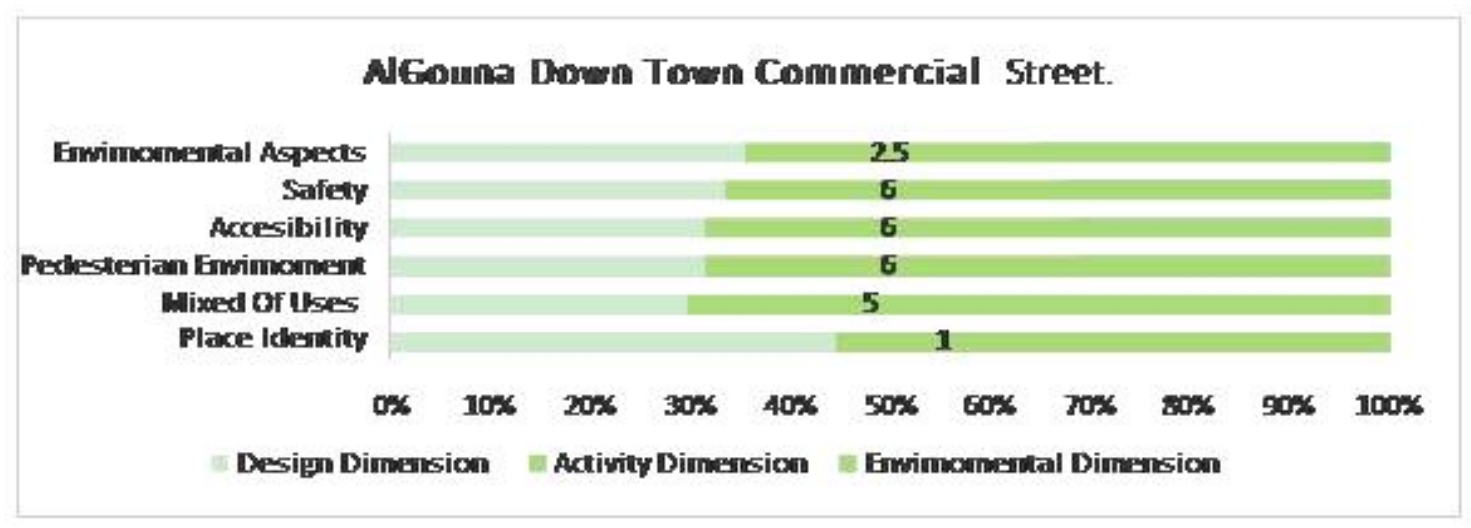

Figure: Chart Shows the Percentage Of sustainable Target in the Street source: The Researcher

In Al Gouna Down Town Street Evaluation Case the state of sustainability is presented in the Six Concepts as shown in the previous figure with a high pedestrian, safety and Comfortable Movement with accessible methods for the street as the toktok where all the users can feel comfort and surrounded by a well-environmental methods and ways to enhance new ways and sustain-durable materials that are used either in buildings and pavements in the street.

Moving to the various activities in the area for shopping and sitting and the street festivals every Sunday which give the street an attractive node for the pedestrians that come to al Downtown Street. The depending of the renewable energy in many fields of the street as lighting and waste management's make the environmental aspects present with moderated Rate

The Defects were clear as the historical identity is not clear as the place is a new place but they are moving on creating a well space identity through the dessert architecture of domes and vaultes to be stored as a different image for this street and to be remembered for even if it's not an origin from AlGouna City 


\section{CONCLUSION}

Regardless of That the Two Studied Examples are in different in context and regions but the referential set got through their strength and weak indicators, therefore the conclusion can be illustrated those Points.

First the six Concepts are found in the Two Different Examples, so this point shows how much the link and the relation between those concepts and the State of sustainability in current sustainable streets and the Concepts Indicators of the referential Set.

Another Conclusion was noticed that although the six concepts were shown in the successful Commercial Streets but in the "Environmental Aspects" are not focused on and a Lack of Environmental Dimension Applications in the Streets, that does not take the importance it should take to keep the Commercial Streets Green and sustainable within the Nature Integration and Enhancing of renewable Resources Technologies.

The Research considered these six concepts as the Concepts of Sustainable Commercial Streets. As they all imperative to reach the state of sustainability, they are: place identity, Mixed of Uses, Pedestrian Environment, Accessibility, Safety and The Environmental Aspects. Coordinating each concept's indicator in design, activity and Environmental Dimensions shaped the Reference table. This table is set into two axials. It consists horizontally from the six concepts and vertically from the three dimensions.

The Referential set was tested on two successful examples, King Victoria and Al gouna Down Town Streets. The six concepts were found together in the two examples; however, they are different contexts and morphology and their cycle of sustainability is different from each example. This confirms and approved the important of those 6 concepts together to reach the sustainable commercial street Target. The two examples achieved minimum of $85 \%$ for the referential set indicators.

\section{REFERENCES}

1. Al-Hagla, Khalid, (2004), Medina Transformation versus Sustaining Identity: Vitality of Medina Genetic Characteristics towards Cultural Sustainability, Alexandria, Egypt: Case Study, Paper presented in: International Seminar "The Mediterranean Medina “"

2. Universita' “G. D’Annunzio" - Facoltà di Architettura. Pescara, Italy

3. Al-Hagla, Khalid, (2005), Sustaining Cultural Identity of the Mediterranean Basin, APJ' Architecture and Planning Journal, Issue No. 16. - Faculty of Architectural Engineering, Beirut Arab University, Beirut, LEBANON.

4. Alexander, C. (2001, 2002, 2005). The Nature of Order: Books 1, 2, and 3, Center for Environmental Structure, Berkeley, California, USA

5. Ahern, J. (1995). Greenways as a Planning Strategy, Landscape and Urban Planning, Vol. 33.

6. Anon., (1997). Measuring Accessibility: An Exploration of issues and Alternatives. Environment and planning, volume A 29, p.75-94

7. Anon., (2012). Pedesteriazation Of Commercial Streets. In: Reclaiming Public Space A Situation Analysis of Commercial Street in Bangalore. India: Evangelical Social Action Forum.

8. Balsas, C.J.K. (2004). Measuring the Livability of an urban Center: an exploratory Study of key Performance Indicators, planning, Practice and Research,pp.101-110

9. Ciolek. (1978). Spatial Behaviour in Pedesterian Areas Ekistics, pp.120-122

10. Ching. (1992). Ordering Principels. In: London, ed. Architecture, form, space and Order Book. s.1.:3rd edition, pp. 339-340.

11. Council, A. D. P. (2006). Abu Dhabi Planning Council 2050. First edition ed. Abudhabi: UAE PLanning council. 
12. Council, A. P., (2004). American Planning Association. In: Chicago: s.n., pp. 245248.

13. Hannallah,Gihan Mosaad R. N. F. (2014). Zero-Carbon Cities as a New Realization of Sustainable Cities. BUE-FISC, p. 124.

14. Government, t. s. (2012). A Myth of Urban Design: The 'Sense of Enclosure' Theory.

15. Jacobs. (1993). Great Streets. s.l.:Cambridge : MA: The MIIT Press..

16. Jacobs, J. (1961). The Uses of Sidewalks. s.l.:s.n.

17. Jones, P. (2008). Creating more people-friendly urban. s.l., s.n.

18. Kaplan, R.K.S. (1989). Environment Preference: A comparison of Four Domains of predictors. Environment and Behavior, pp.21.509-530

19. Kendall, S. (2002). “An Open Building Strategy for Balancing Production Efficiency and Consumer Choice in Housing". Proceedings (vol 1 of 2) 10th Annual Symposium Construction Innovation and Global Competitiveness. University of Cincinnati.Ohio. 